University of Nebraska - Lincoln

DigitalCommons@University of Nebraska - Lincoln

November 1998

\title{
Analysis of multiphoton ionization of metal atoms in the saturation regime using subpicosecond $\mathrm{KrF}$ laser pulses
}

\author{
B. Witzel \\ Laser Chemistry Division, Max-Planck-Institut für Quantenoptik, D-85740 Garching, Federal Republic of \\ Germany \\ Cornelis J. Uiterwaal \\ University of Nebraska - Lincoln, cuiterwaal2@unl.edu \\ H. Schröder \\ Laser Chemistry Division, Max-Planck-Institut für Quantenoptik, D-85740 Garching, Federal Republic of \\ Germany \\ D. Charalambidis \\ Physics Department, University of Crete, Heraklion, Crete, Greece \\ K.-L. Kompa \\ Laser Chemistry Division, Max-Planck-Institut für Quantenoptik, D-85740 Garching, Federal Republic of \\ Germany
}

Follow this and additional works at: https://digitalcommons.unl.edu/physicsuiterwaal

Part of the Physics Commons

Witzel, B.; Uiterwaal, Cornelis J.; Schröder, H.; Charalambidis, D.; and Kompa, K.-L., "Analysis of multiphoton ionization of metal atoms in the saturation regime using subpicosecond KrF laser pulses" (1998). C.J.G.J. Uiterwaal Publications. 8.

https://digitalcommons.unl.edu/physicsuiterwaal/8

This Article is brought to you for free and open access by the Research Papers in Physics and Astronomy at DigitalCommons@University of Nebraska - Lincoln. It has been accepted for inclusion in C.J.G.J. Uiterwaal Publications by an authorized administrator of DigitalCommons@University of Nebraska - Lincoln. 
Phys. Rev. A 58, 3836 - 3848 (1998)

[Issue 5 - November 1998]

\title{
Analysis of multiphoton ionization of metal atoms in the saturation regime using subpicosecond $\mathrm{KrF}$ laser pulses
}

\author{
B. Witzel ${ }^{1}{ }^{*}$, C. J. G. J. Uiterwaal ${ }^{1}{ }^{\dagger}$, H. Schröder ${ }^{1}$, D. Charalambidis ${ }^{2,3}$, and K.-L. \\ Kompa $^{1}$ \\ ${ }^{1}$ Laser Chemistry Division, Max-Planck-Institut für Quantenoptik, P.O. Box 1513, D-85740 \\ Garching, Federal Republic of Germany \\ ${ }^{2}$ Foundation for Research and Technology-Hellas, Institute of Electronic Structure \& Laser, \\ Laser and Applications Division, P.O. Box 1527, GR-711 10 Heraklion, Crete, Greece \\ ${ }^{3}$ Physics Department, University of Crete, Heraklion, Crete, Greece
}

Received 24 March 1998

Singly and multiply charged ion yield curves are reported for resonant and nonresonant twophoton-ionization processes for a collection of 31 metal atoms. The atoms are created by sputtering from a solid target using an Ar-ion gun. Single and multiple ionization of these atoms is performed using linearly polarized 248.6- $\mathrm{nm}(\mathrm{KrF})$ laser pulses with a full width at half maximum duration of $500 \mathrm{fs}$, employing intensities between $10^{9}$ and $10^{12} \mathrm{~W} \mathrm{~cm}^{-2}$. A four-grid high-resolution reflecting time-of-flight spectrometer is used for ion detection. This advanced spectrometer has a well-defined and small source volume, enabling absolute measurements of ionization probabilities and saturation intensities. Because our measurements are not affected by the increase of the interaction volume for increasing intensities, we can discriminate between resonant and nonresonant multiphoton ionization processes without varying the laser pulse duration. For many metals, the intensity dependence of the ion yield can be accurately reproduced by rate calculations based on a resonantly enhanced two-photon-ionization scheme. As a result, we can determine absolute values of the one-photon cross sections in the resonant processes and these are compared to theoretical values we calculated. For the nonresonant processes, we give generalized multiphoton-ionization cross sections and compare these to a scaling law of Lambropoulos [J. Opt. Soc. Am. B 4, 821 (1987)].

(C)1998 The American Physical Society. Used by permission.

URL: http://link.aps.org/abstract/PRA/v58/p3836

DOI: 10.1103/PhysRevA.58.3836

PACS: $32.80 . \mathrm{Rm}$

* Author to whom correspondence should be addressed. Electronic address: KEU@MPQ.MPG.DE

${ }^{\dagger}$ Electronic address: WITZEL@LUCE.IESL.FORTH.GR

[See erratum, attached.] 
Erratum: Analysis of multiphoton ionization of metal atoms in the saturation regime using subpicosecond $\mathrm{KrF}$ laser pulses

[Phys. Rev. A 58, 3836 (1998)]

B. Witzel, ${ }^{*}$ C. J. G. J. Uiterwaal, ${ }^{\dagger}$ H. Schröder, D. Charalambidis, and K.-L. Kompa [S1050-2947(99)04504-7]

PACS number(s): 32.80.Rm, 99.10.+g

Owing to a typesetting error at the final stage of production, the electronic address footnotes for the first two authors were inadvertently interchanged. The correct byline footnotes should read as currently indicated.

*Electronic address: WITZEL@LUCE.IESL.FORTH.GR

${ }^{\dagger}$ Author to whom correspondence should be addressed. Electronic address: KEU@MPQ.MPG.DE 


\title{
Analysis of multiphoton ionization of metal atoms in the saturation regime using subpicosecond KrF laser pulses
}

\author{
B. Witzel, ${ }^{1, *}$ C. J. G. J. Uiterwaal,${ }^{1, \dagger}$ H. Schröder, ${ }^{1}$ D. Charalambidis,${ }^{2,3}$ \\ and K.-L. Kompa ${ }^{1}$ \\ ${ }^{1}$ Laser Chemistry Division, Max-Planck-Institut für Quantenoptik, P.O. Box 1513, D-85740 Garching, Federal Republic of Germany \\ ${ }^{2}$ Foundation for Research and Technology-Hellas, Institute of Electronic Structure \& Laser, \\ Laser and Applications Division, P.O. Box 1527, GR-711 10 Heraklion, Crete, Greece \\ ${ }^{3}$ Physics Department, University of Crete, Heraklion, Crete, Greece
}

(Received 24 March 1998)

\begin{abstract}
Singly and multiply charged ion yield curves are reported for resonant and nonresonant two-photonionization processes for a collection of 31 metal atoms. The atoms are created by sputtering from a solid target using an Ar-ion gun. Single and multiple ionization of these atoms is performed using linearly polarized 248.6-nm $(\mathrm{KrF})$ laser pulses with a full width at half maximum duration of $500 \mathrm{fs}$, employing intensities between $\sim 10^{9}$ and $\sim 10^{12} \mathrm{~W} \mathrm{~cm}^{-2}$. A four-grid high-resolution reflecting time-of-flight spectrometer is used for ion detection. This advanced spectrometer has a well-defined and small source volume, enabling absolute measurements of ionization probabilities and saturation intensities. Because our measurements are not affected by the increase of the interaction volume for increasing intensities, we can discriminate between resonant and nonresonant multiphoton ionization processes without varying the laser pulse duration. For many metals, the intensity dependence of the ion yield can be accurately reproduced by rate calculations based on a resonantly enhanced two-photon-ionization scheme. As a result, we can determine absolute values of the one-photon cross sections in the resonant processes and these are compared to theoretical values we calculated. For the nonresonant processes, we give generalized multiphoton-ionization cross sections and compare these to a scaling law of Lambropoulos [J. Opt. Soc. Am. B 4, 821 (1987)]. [S1050-2947(98)00111-5]
\end{abstract}

PACS number(s): 32.80.Rm

\section{INTRODUCTION}

In this work we present and discuss results on the multiphoton ionization (MPI) of 31 metal atoms. When exposed to a strong electromagnetic field, atoms may undergo MPI [1], in which the atomic system is brought from the ground state into an ionic continuum through the simultaneous absorption of more than one photon. Both resonant and nonresonant ionization schemes will be encountered. Our experimental method consists of two independent steps, namely, (i) sputtering of particles from a solid target by an ion beam and (ii) postionization of the sputtered neutral particles by a laser with fixed frequency. This technique is generally known by its acronyms L-SNMS [2] (laser-secondary neutral mass spectroscopy) or SALI [3] (surface analysis by laser ionization). For a resonant scheme, one also encounters the acronym RIMS, which stands for "resonance ionization mass spectrometry," and can be put in the context of other analytical techniques such as secondary ionization mass spectrometry (SIMS), where one directly studies ions sputtered from a solid target by an ion beam. A great advantage of SALI over SIMS is that the number of sputtered neutral particles is generally two to five orders of magnitude larger than the number of ions, so that an accordingly increased sensitivity might be expected. Furthermore, so-called matrix ef-

\footnotetext{
*Author to whom correspondence should be addressed. Electronic address: KEU@MPQ.MPG.DE

${ }^{\dagger}$ Electronic address: WITZEL@LUCE.IESL.FORTH.GR
}

fects (i.e., effects of the chemical composition of the surface) strongly affect the secondary ion yield in the SIMS method, but in the SALI method this phenomenon is of minor importance. However, as will be shown in this work, matrix effects cannot be neglected in our experiments, as they have an influence on the population of electronically excited states of sputtered neutral atoms.

This paper is organized as follows. In Sec. II the experimental setup is discussed. The experimental data are presented in Sec. III. In Sec. IV we discuss nonresonant MPI (Sec. IV A) and resonantly enhanced MPI (Sec. IV B). For resonantly enhanced MPI, a rate equation model will be presented involving one-photon cross sections. For a selection of typical cases, these cross sections are calculated and compared with the experimental results. Finally, Sec. V is devoted to conclusions.

\section{EXPERIMENT}

The present experiments combine sputtering and laser postionization techniques with a special time-of-flight detection method [4-6] that permits the registration of ions originating exclusively from a so-called confined interaction volume, which is well defined and fixed in space. This powerful combination allows us to perform precise measurements of the intensity dependence of ion yields that are free of volume effects [7] and were not possible hitherto. In the present work the confined interaction volume has rectangular dimensions of $1800 \mu \mathrm{m}$ (along the laser propagation direction) $\times 230 \mu \mathrm{m}$ (along the spectrometer axis) $\times 220 \mu \mathrm{m}$ (volume $V=9.1 \times 10^{-5} \mathrm{~cm}^{3}$ ). The main parts of the setup have been described elsewhere. Therefore, we will only briefly mention 
them here. The laser system [8,9] employed is a hybrid seven-dye double-excimer laser system, producing pulses at a repetition rate of approximately $5 \mathrm{~Hz}$ and with a maximum pulse energy of about $14 \mathrm{~mJ}$. Care was taken to keep the pulse-to-pulse energy fluctuations within a $15 \%$ limit. The system operates at the $\mathrm{KrF}$ excimer wavelength of $248.6 \mathrm{~nm}$ (photon energy $\hbar \omega=4.99 \mathrm{eV}$ ) and has a bandwidth $\Delta \omega_{L}$ of $20 \mathrm{meV}$ or $160 \mathrm{~cm}^{-1}$ that is mainly determined by the gain characteristics of the final $\mathrm{KrF}$ amplifier [8]. The full width at half maximum (FWHM) pulse duration is typically $500 \mathrm{fs}$ and the produced radiation is linearly polarized. To focus the beam, two plano-convex lenses were used with focal lengths of either $f=150$ or $250 \mathrm{~mm}$. The amplified spontaneous emission (ASE) to pulse contrast ratio in the focal plane is better than $10^{-6}$. Sputtering was performed with an Ar-ion gun (Leybold IQE 12/38) mounted at an angle of $45^{\circ}$ with respect to the target surface. The primary ion acceleration voltage was typically $5 \mathrm{kV}$ and typical primary ion currents were $1 \mu \mathrm{A}$. The energy at the target was reduced to $3.5 \mathrm{keV}$ because of the positive repeller voltage that pushes the ions created in the laser postionization towards the ion spectrometer entrance slit. The resolution of the time-of-flight spectrometer was sufficient to resolve the peaks of the isotopes ${ }^{175} \mathrm{Lu}$ and ${ }^{176} \mathrm{Lu}$ (of all metals studied here, $\mathrm{Lu}$ is the one with heaviest pair of consecutive isotopes in reasonable abundances and thus it is the most difficult to resolve).

In the experiments we did not overlap the focus with the confined source volume of the spectrometer, but instead we used the expanding part of the laser beam (behind the focal plane). This approach was chosen to minimize the spatial intensity variation across the confined source volume. It should be noted that owing to spherical aberration the spatial intensity distribution is more homogeneous behind the focal plane than in front of it. Typical distances between the focus and the center of the source volume are $10-15 \mathrm{~mm}$. Being way outside the Rayleigh range, we can use a geometric description of the diverging beam, and assuming a smooth laser profile, we can write the spatiotemporal intensity distribution $I(\mathbf{r}, t)$ inside the confined volume as

$$
I(\mathbf{r}, t)=I_{0} F(t)\left(\frac{f}{z}\right)^{2}
$$

where $I_{0}$ is the unfocused peak intensity (in $\mathrm{W} \mathrm{cm}^{-2}$ ), $f$ the focal length of the lens, $F(t)$ the temporal laser pulse profile (normalized to $F(t=0)=1$; e.g., for a Gaussian pulse $F(t)$ $=\exp \left[-(\ln 2)(t / \tau)^{2}\right]$, with $2 \tau$ the FWHM duration of the pulse), and $z$ the distance from the focal plane along the laser propagation direction. For the space-averaged value $I_{\text {av }}$ of the local peak intensity in the source volume we find

$$
I_{\mathrm{av}}=I_{0} \frac{f^{2}}{d^{2}-b^{2}},
$$

where $d$ is the distance between the focal plane and the center of the rectangular confined volume and $2 b$ the size of this volume along the laser direction. This implies that the maximum and minimum values of the peak intensity occurring in this volume are given by $I_{\max } / I_{\mathrm{av}}=(d+b) /(d-b)$ and $I_{\min } / I_{\mathrm{av}}=(d-b) /(d+b)$, respectively. Thus, for $b \ll d$, the relative spatial intensity variation is $2 b / d$ and in order to make this variation as small as possible it seems advantageous to make $d$ as large as possible. However, Eq. (2) shows that this will at the same time decrease the available intensity and thus a compromise has to be sought. In the experiments, we always chose $d$ to be between 10 and 15 $\mathrm{mm}$ so that the relative spatial variation of the intensity was between $12 \%$ and $18 \%$ and thus comparable to the intrinsic pulse-to-pulse energy fluctuation of the laser system. For the used values of $d$, only $2-12 \%$ of the total laser pulse energy actually flows through the confined volume. The unfocused intensity appearing in Eq. (2) is experimentally determined using $I_{0}=E_{p} / A_{0} \Delta t$, where $E_{p}$ is the measured value of the pulse energy, $A_{0}=10 \times 27 \mathrm{~mm}^{2}$ is the area of the unfocused beam spot, and $\Delta t=\int F\left(t^{\prime}\right) d t^{\prime}=532.2$ fs is the width of the rectangular pulse profile that has the same energy as a Gaussian pulse profile with a FWHM of 500 fs. To attenuate the laser beam, we use a pair of dielectric plates with an angledependent attenuation covering three orders of magnitude. By rotating the plates over equal but opposite angles no net beam displacement results. The pulse energy $E_{p}$ was measured using a pyroelectric detector. Energy measurements were performed by averaging over at least ten laser pulses immediately before and after each ion-yield measurement. We will plot our measured ion yields as a function of $I_{\mathrm{av}}$, calculated with Eq. (2). The estimated uncertainty in the intensity is about $30 \%$. Typical intensities employed range from $10^{9}$ to $10^{12} \mathrm{~W} \mathrm{~cm}^{-2}$ and are thus comparable to intensities prevalent in the focus of a common nanosecond excimer laser. To avoid serious window fogging (decrease of the transmission as a result of deposits) we regularly replaced the entrance window. To keep an eye on the decrease in transmission during the progress of the experiments and to be able to correct for this effect, we performed intensity calibration measurements just before and after the replacement of each window, as well as several times in between.

After their flight through the spectrometer, the ions were detected using a double multichannel plate detector, a preamplifier, and a 500-MHz digitizing oscilloscope (Tektronix Inc., model No. TDS 644A). In some cases, for calibration purposes, ions were counted using a $2-\mathrm{GHz}$ multiscaler (FAST ComTec, Germany, model No. 7886). Raw data were stored in a personal computer where they were further processed. The solid metallic targets consisted of flat pieces of foil that were screwed on the repeller electrode and thus carried the repeller voltage during the experiment. All metallic targets were commercially obtained from Goodfellow Ltd. (Cambridge, United Kingdom) in the purest grade available (always $\geqslant 99.9 \%$ ) and used without further treatment.

\section{RESULTS}

MPI yields in the intensity range from $10^{9}$ to $10^{12} \mathrm{~W} \mathrm{~cm}{ }^{-2}$ were investigated for the following 31 metals: $\mathrm{Mg}, \mathrm{Al}, \mathrm{Sc}, \mathrm{Fe}, \mathrm{Co}, \mathrm{Ni}, \mathrm{Cu}, \mathrm{Zn}, \mathrm{Nb}, \mathrm{Rh}, \mathrm{Ag}, \mathrm{Cd}$, In, Pr, Sm, $\mathrm{Eu}, \mathrm{Gd}, \mathrm{Tb}, \mathrm{Dy}, \mathrm{Ho}, \mathrm{Er}, \mathrm{Tm}, \mathrm{Yb}, \mathrm{Lu}, \mathrm{Ta}, \mathrm{W}, \mathrm{Re}, \mathrm{Au}, \mathrm{Bi}, \mathrm{Th}$, and $\mathrm{U}$. These can be subdivided according to their groundstate electron configurations as follows: one metal from group IIa (alkali-earth metals) with an $s^{2}$ outer shell $(\mathrm{Mg})$, two metals from group IIIa with $s^{2}\left(d^{10}\right) p$ outer shells (Al and In), one metal from group Va with a $p^{3}$ outer shell (Bi), six metals from the first transition (or iron) group with 


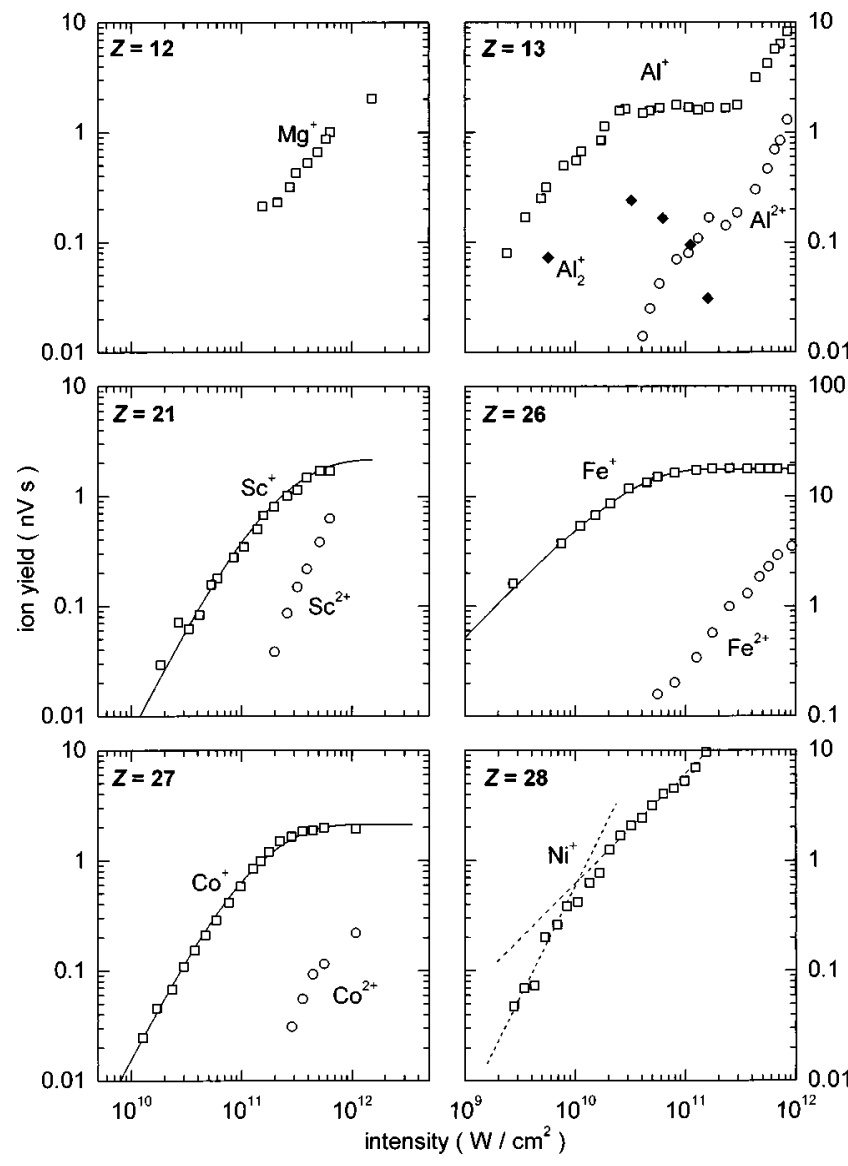

FIG. 1. Measured ion yields as a function of laser intensity for $\operatorname{Mg}(Z=12), \operatorname{Al}(Z=13), \operatorname{Sc}(Z=21), \mathrm{Fe}(Z=26)$, Co $(Z=27)$, and $\mathrm{Ni}(Z=28)$.

$4 s^{x} 3 d^{y}$ outer subshells ( $\mathrm{Sc}, \mathrm{Fe}, \mathrm{Co}, \mathrm{Ni}, \mathrm{Cu}$, and $\mathrm{Zn}$ ), four metals from the second transition (or palladium) group with $5 s^{x} 4 d^{y}$ outer subshells ( $\mathrm{Nb}, \mathrm{Rh}, \mathrm{Ag}$, and $\mathrm{Cd}$ ), four metals from the third transition (or platinum) group with $6 s^{x} 5 d^{y}$ outer subshells ( $\mathrm{Ta}, \mathrm{W}, \mathrm{Re}$, and $\mathrm{Au}$ ), eleven lanthanides, or rare-earth elements, with incomplete $4 f$ and $5 d$ subshells (Pr, Sm, Eu, Gd, Tb, Dy, Ho, Er, Tm, Yb, and Lu), and two actinides with incomplete $5 f$ and $6 d$ subshells (Th and $\mathrm{U}$ ). For calibration purposes, in particular to ensure the correct intensity dependences below saturation, we also investigated the molecules $\mathrm{SO}_{2}$ and NO. Our results are compiled in Figs. $1-6$ in a $\log -\log$ representation. Some of the data curves may seem to look rather peculiar at first sight. However, we wish to emphasize that to the best of our knowledge they reflect real and sometimes complicated ionization processes of sputtered entities. In this paper we will mainly discuss the singly charged ion yields, although higher charge states were observed as well: Doubly charged ions were observed for all metals with the exception of $\mathrm{Mg}, \mathrm{Ni}, \mathrm{Zn}$, and $\mathrm{Cd}$, and for $\mathrm{Ho}$, $\mathrm{Tm}, \mathrm{Lu}$, and $\mathrm{Ta}$ we even observed triply charged ions. In some cases, ionized dimers were observed as well as metal oxides and, in one single case, also a metal nitride $\left(\mathrm{NbN}^{+}\right)$. A quantitative analysis of the yields of higher charge states and of molecular ions would require additional calibrations (measurements of the relative detection efficiencies) that we have not yet performed. Therefore, we show all ion yields as measured, i.e., not corrected for detection efficiencies.

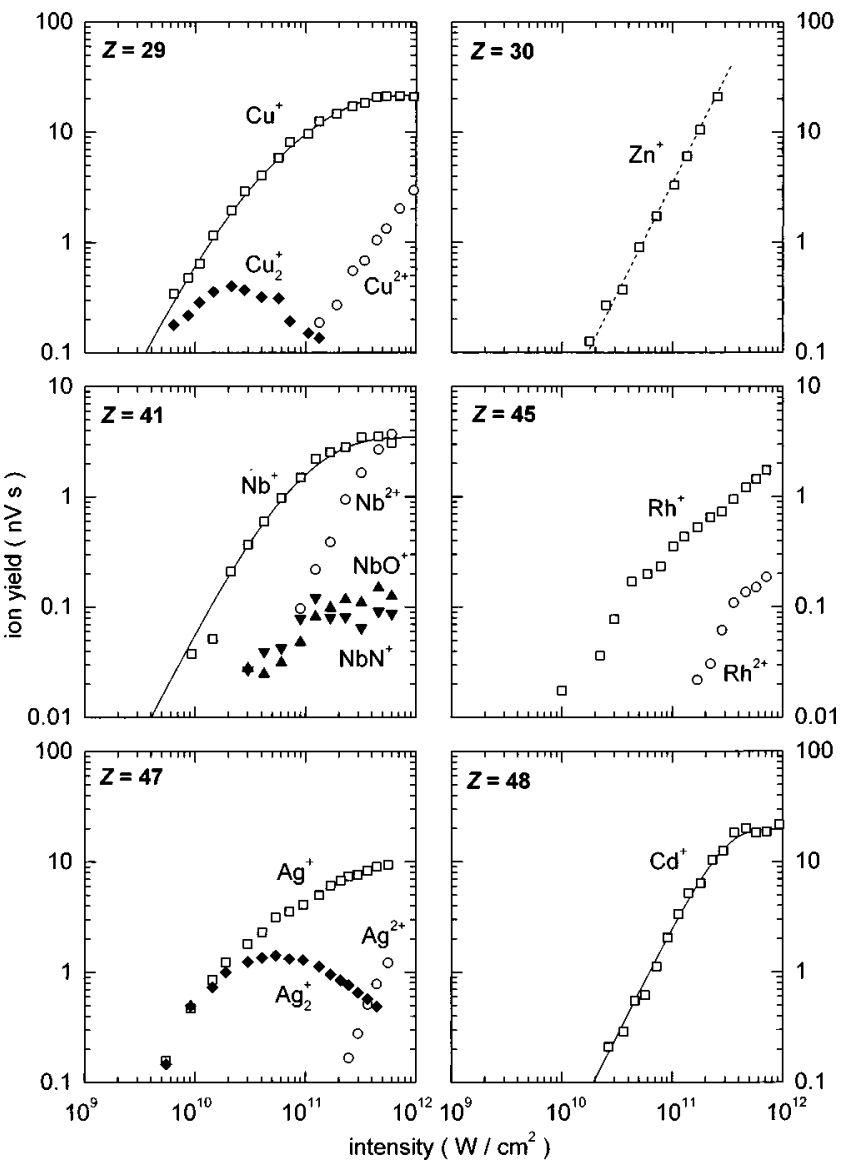

FIG. 2. Measured ion yields as a function of laser intensity for $\mathrm{Cu}(Z=29), \mathrm{Zn}(Z=30), \mathrm{Nb}(Z=41), \mathrm{Rh}(Z=45), \mathrm{Ag}(Z$ $=47)$, and $\mathrm{Cd}(Z=48)$.

\section{DISCUSSION}

Inspecting the singly charged ion yield curves shown in Figs. 1-6, one immediately notices that the curves for the two group IIb elements $\mathrm{Zn}(Z=30)$ and $\mathrm{Cd}(Z=48)$ are somewhat exceptional: For a large range of intensities, they exhibit a clear unperturbed quadratic intensity dependence. In the case of $\mathrm{Cd}$, saturation is reached at an intensity of $(2.6 \pm 0.4) \times 10^{11} \mathrm{~W} \mathrm{~cm}^{-2}$; the curve sharply bends here and reaches the saturation plateau. This sharp bending of the $\mathrm{Cd}$ curve makes it unique among all measured curves. Below we will show that $\mathrm{Zn}$ and $\mathrm{Cd}$ are in fact ionized in a two-photon nonresonant MPI process, whereas in all other cases the ionization is resonantly enhanced. Therefore, our discussion will proceed as follows. In Sec. IV A we will treat nonresonant MPI on the basis of a scaling law and estimate upper limits for nonresonant saturation intensities. We will then make it plausible that ac Stark shifts of intermediate atomic energy levels in the atomic spectra are negligible, even for the overestimated saturation intensities the scaling law yields. We will then discuss the exceptional cases of $\mathrm{Zn}$ and $\mathrm{Cd}$. In Sec. IV B we will deal with resonantly enhanced MPI. After the presentation of a simple rate equation model we will also demonstrate how the one-photon cross sections appearing in this model can be calculated. For a selection of metals we will compare our experimental results with this model. In Sec. IV C we will make some final remarks. 


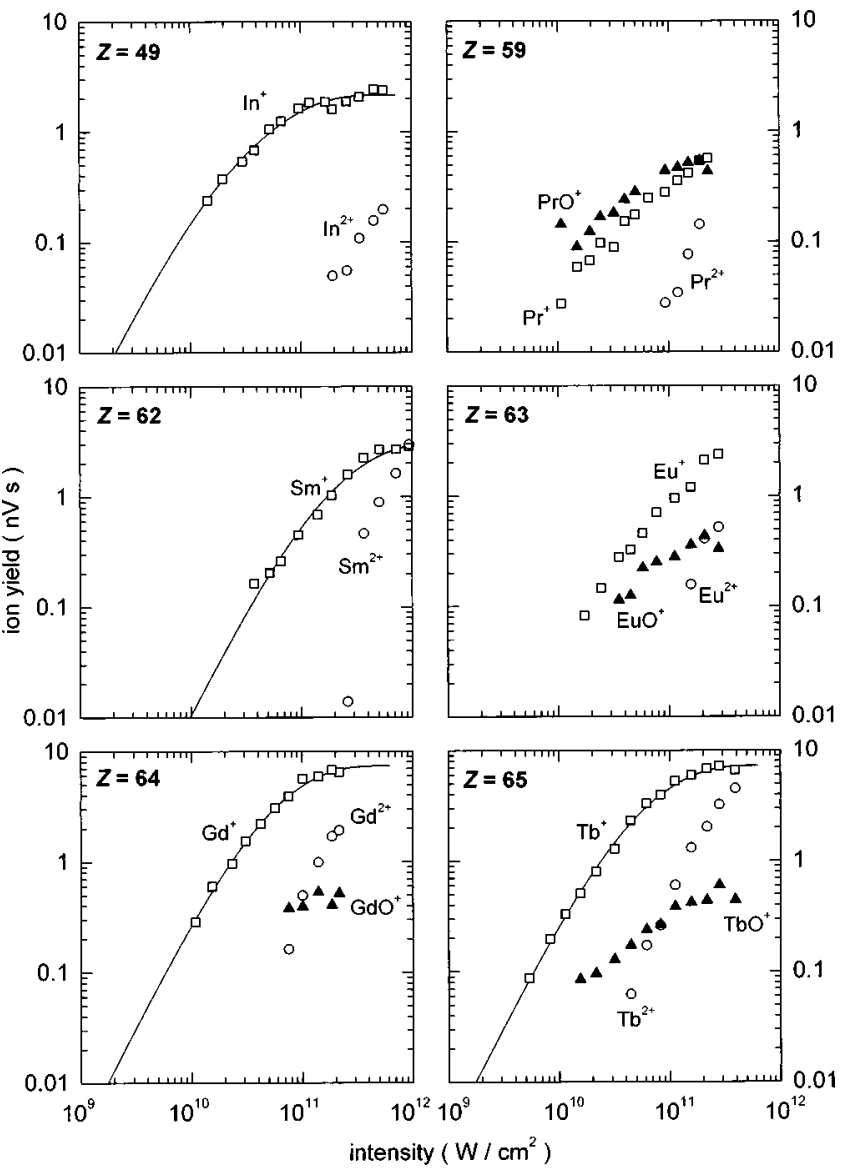

FIG. 3. Measured ion yields as a function of laser intensity for In $(Z=49), \operatorname{Pr}(Z=59), \operatorname{Sm}(Z=62), \operatorname{Eu}(Z=63), \mathrm{Gd}(Z=64)$, and $\mathrm{Tb}(Z=65)$.

\section{A. Nonresonant MPI, scaling law, and ac Stark shifts}

For the atoms investigated here, the lowest ionization potentials range from $5.42 \mathrm{eV}$ (for Pr) to $9.394 \mathrm{eV}$ (for $\mathrm{Zn}$ ). Therefore, lowest-order perturbation theory [10] (LOPT) predicts nonresonant ionization from the ground state into the lowest continuum to be a two-photon process. The ionization rate $d P / d t\left(\mathrm{~s}^{-1}\right)$ is then given by

$$
\frac{d P}{d t}(t)=[1-P(t)] \sigma_{(2)} \Phi^{2}(t),
$$

where $P(t)$ is the probability to find the atom ionized at time $t, \sigma_{(2)}$ is the generalized two-photon-ionization cross section (in $\mathrm{cm}^{4} \mathrm{~s}$ ) that in general is intensity and thus time dependent (e.g., due to level shifting), and $\Phi(t)$ is the time-dependent photon flux (in $\mathrm{cm}^{-2} \mathrm{~s}^{-1}$ ). For the special case of a generalized ionization cross section that does not depend on intensity, we can find a simple expression for the ionization probability. Using the normalized laser temporal profile $F(t)$ introduced in Eq. (1) and writing $\Phi_{0}=I_{0} / \hbar \omega$ for the maximum photon flux (occurring at $t=0$ ), we find from Eq. (3) the ionization probability after the pulse is completed (so for $t \rightarrow \infty)$ :

$$
P(t \rightarrow \infty)=1-e^{-\left(I_{0} / I_{\mathrm{sat}}\right)^{2}}
$$

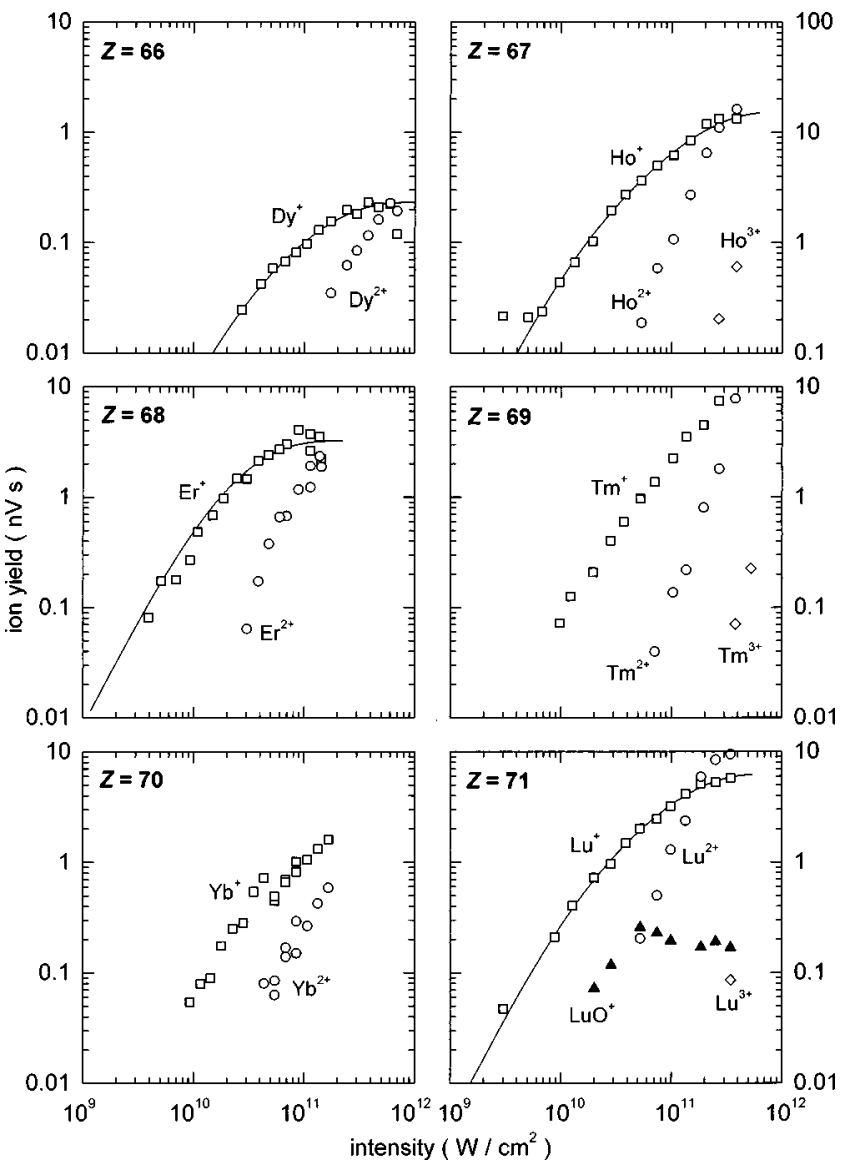

FIG. 4. Measured ion yields as a function of laser intensity for Dy $(Z=66)$, Ho $(Z=67)$, Er $(Z=68)$, Tm $(Z=69)$, Yb $(Z$ $=70)$, and $\mathrm{Lu}(Z=71)$.

in which we have introduced the saturation intensity $I_{\text {sat }}$, defined by

$$
I_{\mathrm{sat}}=\frac{\hbar \omega}{\sqrt{\sigma_{(2)} t_{\mathrm{eff}}}} .
$$

The effective pulse duration appearing in the latter formula is given by $t_{\text {eff }}=\int_{-\infty}^{\infty} F^{2}\left(t^{\prime}\right) d t^{\prime}$. For a Gaussian temporal pulse shape with a FWHM duration of $500 \mathrm{fs}, t_{\text {eff }} \approx 376.3 \mathrm{fs}$. Neglecting volume effects and assuming that processes involving doubly, triply, etc., ionized and/or other species do not interfer, we see from Eq. (4) that a $\log$-log plot of $P(t$ $\rightarrow \infty)$ vs peak intensity $I_{0}$ has two straight asymptotes, namely, $P(t \rightarrow \infty)=\left(I_{0} / I_{\text {sat }}\right)^{2}$ for $I_{0} \ll I_{\text {sat }}$ and $P(t \rightarrow \infty)=1$ for $I_{0} \gg I_{\text {sat }}$, i.e., for saturation [11]. Experimentally, the value of $I_{\text {sat }}$, which contains all information on the process, can in principle be determined from the abscissa value of the intersection point of the two asymptotes. To get an idea of the order of magnitude of the saturation intensity, we make use of a scaling law [10] that estimates $\sigma_{(2)}^{A}$ for an atom $A$ on the basis of the known value of the two-photon-ionization cross section $\sigma_{(2)}^{\mathrm{H}}$ for atomic hydrogen. Writing $\sigma_{(2)}$ $=\left(\Lambda_{(2)}\right)^{2}$, this estimation is given by

$$
\Lambda_{(2)}^{A}=\Lambda_{(2)}^{\mathrm{H}} \frac{R_{A}^{2} E_{\infty}^{\mathrm{H}}}{R_{\mathrm{H}}^{2} E_{\infty}^{A}},
$$



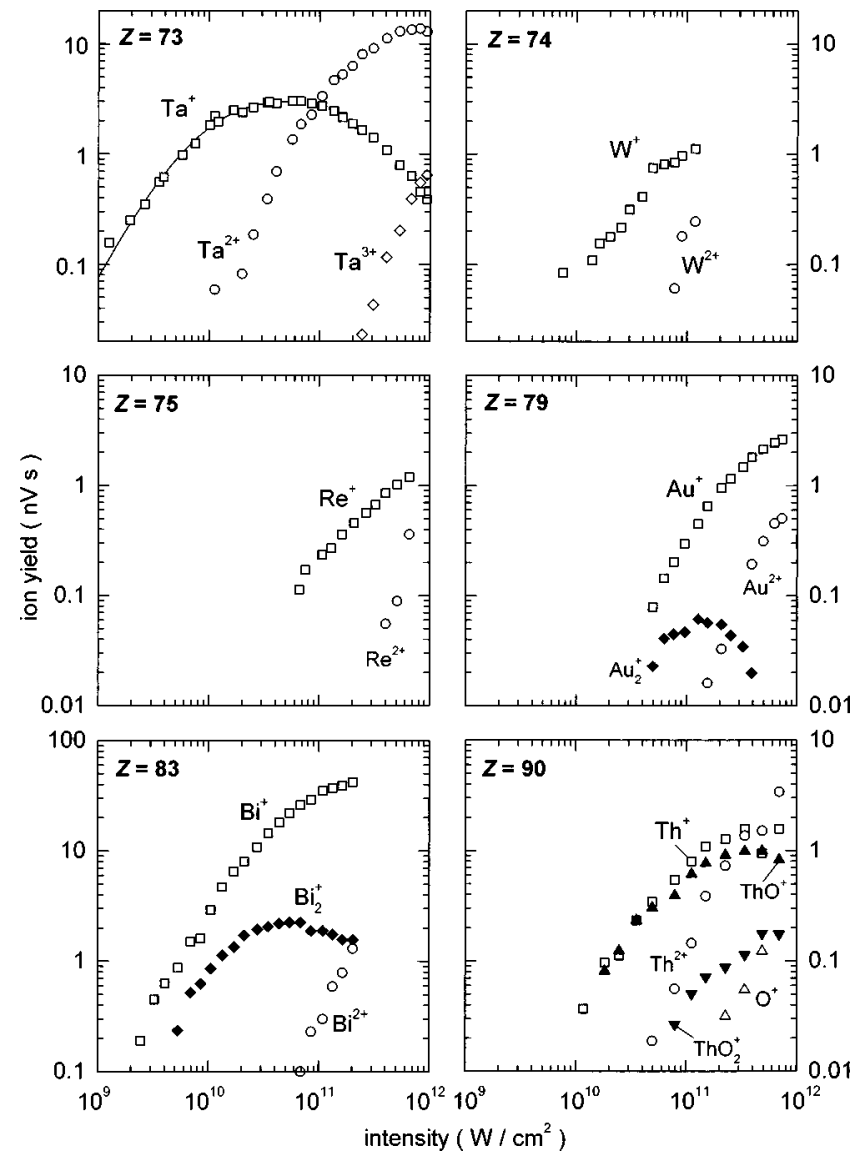

FIG. 5. Measured ion yields as a function of laser intensity for Ta $(Z=73), \mathrm{W}(Z=74), \operatorname{Re}(Z=75), \mathrm{Au}(Z=79), \mathrm{Bi}(Z=83)$, and $\mathrm{Th}(Z=90)$.

where $R$ is the atomic radius, $E_{\infty}$ is the ionization potential, and $\Lambda_{(2)}^{\mathrm{H}}=10^{-26} \mathrm{~cm}^{2} \mathrm{~s}^{1 / 2}$. Equation (6) underestimates the generalized cross section and thus overestimates the saturation intensity because resonances with intermediate atomic states have not been taken into account. Using this scaling

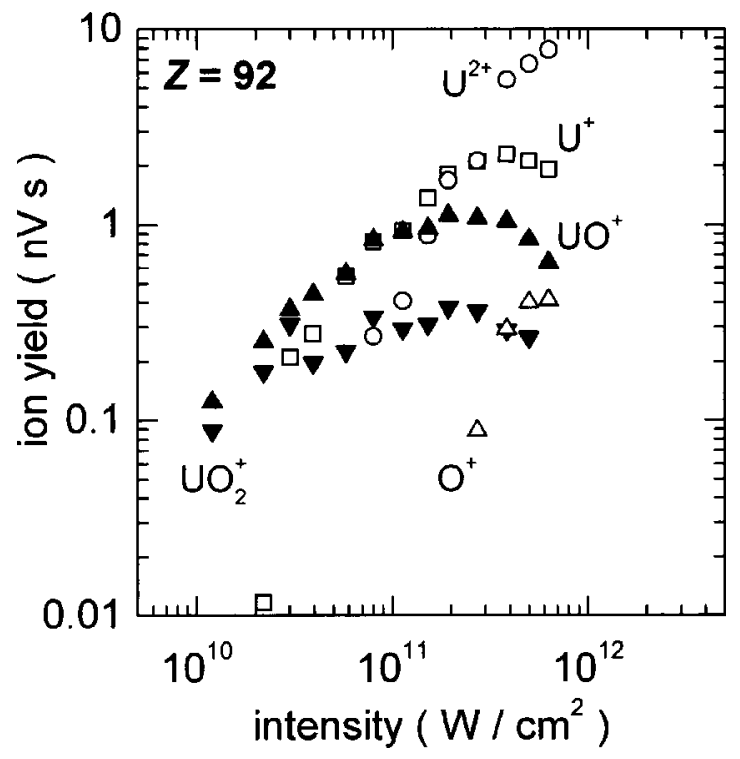

FIG. 6. Measured ion yields as a function of laser intensity for $\mathrm{U}$ $(Z=92)$. model, we can now estimate all relevant saturation intensities. They range between $3.7 \times 10^{12} \mathrm{~W} \mathrm{~cm}^{-2}$ (for Eu) and $1.5 \times 10^{13} \mathrm{~W} \mathrm{~cm}^{-2}$ (for $\mathrm{Fe}$ ). These values can now be used to estimate the ponderomotive shift, given (in a.u.) by [12]

$$
U_{P}=\frac{E^{2}}{4 \omega^{2}}=\frac{2 \pi \alpha}{\omega^{2}} I
$$

where $\omega$ is the field angular frequency, $E$ is the electric field strength, and $\alpha$ is the fine-structure constant. In practical units and for $\lambda=248.6 \mathrm{~nm}$ (i.e., $\omega=0.1833$ a.u.), Eq. (7) becomes

$$
U_{P}(\mathrm{eV})=5.771 \times 10^{-15} I\left(\mathrm{~W} \mathrm{~cm}^{-2}\right)
$$

We thus see that for all atoms considered here, the ponderomotive shift at the saturation intensity is between 21 and 83 $\mathrm{meV}$. The scaling model has been derived having in mind processes involving typically much more than two photons. Therefore, the saturation intensity values it produces for a two-photon process are perhaps not very accurate. In addition, as mentioned before, it generally overestimates the saturation intensity. However, the argumentation given here serves only to demonstrate that ac Stark shifts in the atomic spectra are relatively minor and we will therefore neglect them. (We assume that the ac Stark shift of the bound states is of the same order of magnitude as the ponderomotive shift of the ionization threshold.) This further implies that dynamical resonances [13] are not of any importance for intensities below and around the saturation intensity and we can thus maintain the convenient picture of an intensityindependent atomic structure and thus of an intensityindependent generalized ionization cross section.

We will now discuss $\mathrm{Zn}$ and $\mathrm{Cd}$. For these two atoms, the closest dipole-allowed intermediate states are 430 and 808 $\mathrm{meV}$ away, respectively, from the one-photon energy level and thus we do not expect a resonantly enhanced ionization process. In our Zn measurements, a quadratic intensity dependence (see the dashed line in Fig. 2) is observed over an intensity range that is more than a decade wide, and even for the highest intensity of about $2.5 \times 10^{11} \mathrm{~W} \mathrm{~cm}^{-2}$ we do not

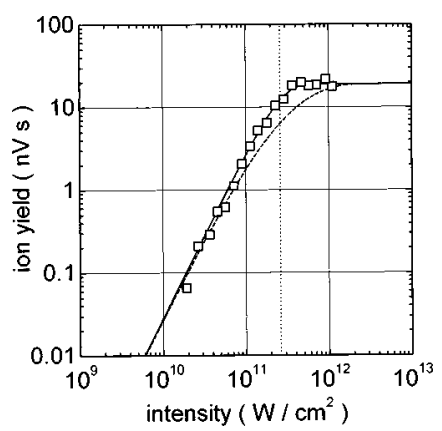

FIG. 7. Measured MPI yields of $\mathrm{Cd}$ as a function of intensity. The solid curve is a fit based on Eq. (14). The dotted line indicates the saturation intensity. For comparison, an ion-yield curve based on Eq. (15) for a two-step resonantly enhanced MPI scheme is shown (dashed curve) that has the same asymptotic behavior for the low- and high-intensity limits. For this curve, we took $\sigma_{2} / 2 \sigma_{1}=1$, making the twist in the resonant curve as sharp as possible. Clearly, MPI in Cd is nonresonant. 
TABLE I. Experimental ( $\left.\sigma_{1, \text { expt }}, \sigma_{2, \text { expt }}\right)$ and calculated $\left(\sigma_{1, \text { calc }}\right)$ values of the resonantly enhanced MPI cross sections for Sc, Fe, Co, Sm, Dy, Ho, Er, and Ta. For each of the listed pairs $\left(\sigma_{1}, \sigma_{2}\right)$ there exists another pair $\left(\frac{1}{2} \sigma_{2}, 2 \sigma_{1}\right)$ that gives an equally good fit to the experimental data (see the text). The errors given are standard errors and include the experimental uncertainty in the laser intensity. For Sc, $\mathrm{Fe}, \mathrm{Co}$, and $\mathrm{Ta}$, we give the largest calculated cross section starting from any state in the ground-state multiplet (see the text) as well as the transition for which this cross section is found. (For Ta, the configuration of the intermediate resonant state is not well known.) For Cd, an experimental and a theoretical value of the generalized two-photon cross section are given in the bottom row.

\begin{tabular}{|c|c|c|c|c|}
\hline Element & $\begin{array}{c}\sigma_{1, \mathrm{expt}} \\
\left(10^{-17} \mathrm{~cm}^{2}\right)\end{array}$ & $\begin{array}{c}\sigma_{2, \mathrm{expt}} \\
\left(10^{-17} \mathrm{~cm}^{2}\right)\end{array}$ & $\begin{array}{c}\text { Largest } \sigma_{1, \text { calc }} \\
\left(10^{-17} \mathrm{~cm}^{2}\right)\end{array}$ & Transition with largest $\sigma_{1, \text { calc }}$ \\
\hline $\operatorname{Sc}(Z=21)$ & $1.6 \pm 0.8$ & $1.0 \pm 0.5$ & 1.005 & $\begin{array}{l}3 d 4 s^{2} a^{2} D_{5 / 2}\left(168.34 \mathrm{~cm}^{-1}\right) \rightarrow \\
\quad 3 d 4 s\left({ }^{3} D\right) 5 p{ }^{2} D_{5 / 2}^{o}\left(40351.30 \mathrm{~cm}^{-1}\right) \\
\quad\left(\text { for } M= \pm \frac{5}{2}\right)\end{array}$ \\
\hline $\mathrm{Fe}(Z=26)$ & $460 \pm 240$ & $4.9 \pm 1.6$ & 405.1 & $\begin{array}{l}3 d^{6} 4 s^{2} a^{5} D_{3}\left(415.932 \mathrm{~cm}^{-1}\right) \rightarrow \\
\quad 3 d^{6}\left({ }^{5} D\right) 4 s 4 p\left({ }^{1} P^{o}\right) x^{5} F_{4}^{o}\left(40594.429 \mathrm{~cm}^{-1}\right) \\
\quad(\text { for } M=0)\end{array}$ \\
\hline $\operatorname{Co}(Z=27)$ & $1.5 \pm 0.5$ & $2.9 \pm 1.0$ & 2.549 & $\begin{array}{l}3 d^{7} 4 s^{2} a^{4} F_{3 / 2}\left(1809.33 \mathrm{~cm}^{-1}\right) \rightarrow \\
\quad 3 d^{7} 4 s\left(a^{5} P\right) 4 p z^{4} P_{1 / 2}^{o}\left(41969.90 \mathrm{~cm}^{-1}\right) \\
\quad\left(\text { for } M= \pm \frac{1}{2}\right)\end{array}$ \\
\hline $\operatorname{Sm}(Z=62)$ & $0.84_{-0.75}^{+0.63}$ & $1.7_{-0.8}^{+5.6}$ & & \\
\hline Dy $(Z=66)$ & $1.0 \pm 0.4$ & $14 \pm 8$ & & \\
\hline Ho $(Z=67)$ & $0.9 \pm 0.3$ & $26 \pm 12$ & & \\
\hline $\operatorname{Er}(Z=68)$ & $8 \pm 3$ & $17 \pm 5.5$ & & \\
\hline \multirow[t]{2}{*}{ Ta $(Z=73)$} & $30_{-15}^{+80}$ & $60_{-30}^{+160}$ & 65.17 & $\begin{array}{l}5 d^{3} 6 s^{2} a^{4} F_{3 / 2}(\text { ground state }) \rightarrow \\
\quad ? J=\frac{3}{2}\left(40230.01 \mathrm{~cm}^{-1}\right) \\
\quad\left(\text { for } M= \pm \frac{3}{2}\right)\end{array}$ \\
\hline & $\sigma_{(2), \operatorname{expt}}$ & & $\sigma_{(2), \text { calc }}$ & \\
\hline Element & $\left(10^{-47} \mathrm{~cm}^{4} \mathrm{~s}\right)$ & & $\left(10^{-47} \mathrm{~cm}^{4} \mathrm{~s}\right)$ & \\
\hline $\mathrm{Cd}(Z=48)$ & $2.5 \pm 0.8$ & & $1.6 \times 10^{-3}$ & \\
\hline
\end{tabular}

observe any onset of saturation, so that the saturation intensity of $\mathrm{Zn}$ must be substantially higher. In Fig. 7 we show the measured intensity dependence of the $\mathrm{Cd}^{+}$yield together with a best fit according to Eq. (4) (solid curve). The other (dashed) curve in Fig. 7 is a "limiting" resonantly enhanced MPI ion-yield curve that will be discussed below. Clearly, MPI of Cd is a nonresonant process. The saturation intensity of $\mathrm{Cd}$ (indicated by a dotted line in Fig. 7) is (2.6 \pm 0.4 ) $\times 10^{11} \mathrm{~W} \mathrm{~cm}^{-2}$; it corresponds to a generalized two-photonionization cross section of $(2.5 \pm 0.8) \times 10^{-47} \mathrm{~cm}^{4} \mathrm{~s}$ (see Table I). Using the scaling law, we find $I_{\text {sat,Cd }} \approx 1.0$ $\times 10^{13} \mathrm{~W} \mathrm{~cm}^{-2}$ and $I_{\mathrm{sat}, Z \mathrm{n}} \approx 1.3 \times 10^{13} \mathrm{~W} \mathrm{~cm}^{-2}$. Thus the theoretical value for $\mathrm{Cd}$ is 38 times larger than what we observe experimentally. On the other hand, the scaling law model correctly predicts $\mathrm{Zn}$ to have a higher saturation intensity than Cd. Because this model is known to overestimate saturation intensities, it is not at variance with our experimental results. It must be noticed that the applicability of the scaling law can be rationalized, e.g., by inspecting the LOPT expression for the generalized $K$-photon-ionization cross section $\sigma_{(K)}$ and noticing that for large numbers of photons, say, $K=10$ or more, $\sigma_{(K)}^{1 / K}$ scales proportionally to the atomic size and inversely proportionally to the ionization potential. This conclusion can be arrived at by replacing the detunings and dipole matrix elements appearing in the LOPT expression by appropriate average values and inspecting the behavior of such average values for large numbers of photons. Therefore, in a sense, the scaling law has a statistical aspect and we can anticipate its predictions to be off for $\mathrm{Cd}$ and $\mathrm{Zn}$, where the atom is ionized by just two photons, which clearly is not a large number.

For all atoms considered here, a higher saturation intensity than for $\mathrm{Zn}$ is predicted by the scaling law only for $\mathrm{Co}\left(I_{\mathrm{sat}, \mathrm{Co}} \approx 1.3 \times 10^{13} \mathrm{~W} \mathrm{~cm}^{-2}\right)$ and $\mathrm{Fe}\left(I_{\mathrm{sat}, \mathrm{Fe}} \approx 1.5\right.$ $\times 10^{13} \mathrm{~W} \mathrm{~cm}^{-2}$ ), but for these atoms the saturation intensity is significantly lowered because of resonant ionization. This is well known for Fe [4]. For Co several allowed transitions can be found [14] close to the laser wavelength (some of which start from a low-lying excited state, which may get populated as a result of the sputtering process). In the following subsection these two (and other) atoms will be discussed in further detail.

\section{B. Resonantly enhanced MPI and rate equation calculations}

The ion-yield curves for all other atoms are qualitatively and quantitatively different from the nonresonant MPI curves 
observed for $\mathrm{Zn}$ and $\mathrm{Cd}$. In particular, when reaching a saturation plateau, the curves never bend as sharply as in the $\mathrm{Cd}$ case. Furthermore, a slope close to one is encountered for many atoms over a wide range of intensities. For Fe, this unity slope is found even for the lowest intensities used of about $10^{9} \mathrm{~W} \mathrm{~cm}^{-2}$. All this clearly suggests that ionization in most cases proceeds via a $(1+1)$ resonantly enhanced MPI (REMPI) scheme. For a more detailed analysis, we have performed rate equation calculations and compared the results with our experimental data. This procedure, however, could not be consistently applied in all cases. In our spectra for $\mathrm{Al}, \mathrm{Cu}, \mathrm{Nb}, \mathrm{Ag}, \mathrm{Pr}, \mathrm{Eu}, \mathrm{Gd}, \mathrm{Tb}, \mathrm{Lu}, \mathrm{Au}, \mathrm{Bi}, \mathrm{Th}$, and $\mathrm{U}$ targets, we observe molecular ions in addition to the atomic ions. This implies that ions may now be created from different parent species. The slope observed then also reflects dissociative ionization of a precursor molecule instead of only atomic ionization. This effect is well known, e.g., for $\mathrm{Cu}$ [15]. Remarkably, we observe a dimer-ion signal (see Figs. 1-6) not only for $\mathrm{Cu}$, but for all three elements of group $\mathrm{Ib}$, including silver $(\mathrm{Ag})$ and gold $(\mathrm{Au})$. In addition, dimer ions are found for $\mathrm{Al}$ and $\mathrm{Bi}$. In fact, for these five metals, the singly ionized dimers were the only detectable molecules. For the other metals in this category, the molecules produced were all oxides, namely, $\mathrm{NbO}^{+}, \mathrm{PrO}^{+}, \mathrm{EuO}^{+}$, $\mathrm{GdO}^{+}, \mathrm{TbO}^{+}, \mathrm{LuO}^{+}, \mathrm{ThO}^{+}, \mathrm{ThO}_{2}^{+}, \mathrm{UO}^{+}$, and $\mathrm{UO}_{2}^{+}$; in one case a nitride was observed $\left(\mathrm{NbN}^{+}\right)$. In the experiments with Th and $\mathrm{U}$, we also observed a considerable $\mathrm{O}^{+}$signal. Remarkably, oxides are found only for lanthanides and actinides (with the exception of $\mathrm{Nb}$ ) and oxides and dimers are never found simultaneously. Here we do not further discuss these observations because they are related to the sputtering process itself; for a valuable review on sputtering the reader is referred, e.g., to Ref. [16]. The ion yield curves for Mg, $\mathrm{Rh}, \mathrm{In}, \mathrm{Tm}, \mathrm{Yb}, \mathrm{W}$, and Re are more or less anomalous and these will be very briefly discussed in Sec. IV C.

We will now describe our rate equation calculations. The rate equation approach is justified by the fact that the coherence time $\tau_{\text {coh }}$ of our laser as given by [17] $\tau_{\text {coh }}=2 / \Delta \omega_{L}$ amounts to about $50 \mathrm{fs}$ and thus it is only a small fraction of the total laser pulse duration of $500 \mathrm{fs}$. Our rate equation calculations are based on a ladder scheme, in which we label the ground state of the metal atom with 1 , the intermediate (and possibly but not necessarily resonant) state with 2 , and the upper state, corresponding to the ionized system, with 3. The rate equations are then

$$
\begin{aligned}
\frac{d n_{1}(t)}{d t}= & -\sigma_{1} \Phi(t) n_{1}(t)+k n_{2}(t)+\sigma_{1} \Phi(t) n_{2}(t), \\
\frac{d n_{2}(t)}{d t}= & +\sigma_{1} \Phi(t) n_{1}(t)-k n_{2}(t) \\
& -\sigma_{1} \Phi(t) n_{2}(t)-\sigma_{2} \Phi(t) n_{2}(t), \\
\frac{d n_{3}(t)}{d t}= & +\sigma_{2} \Phi(t) n_{2}(t) .
\end{aligned}
$$

Here $n_{i}(t)$ denotes the population of level $i, \sigma_{1}$ and $\sigma_{2}$ are the one-photon cross sections for the $1 \rightarrow 2$ and the $2 \rightarrow 3$ transitions, respectively, $\Phi(t)$ is the time-dependent photon flux (number of photons per unit area per unit time), and $k$ is the relaxation rate of the intermediate level. Values for $k$ are typically between $10^{8} \mathrm{~s}^{-1}$ (for a one-photon allowed intermediate state) and $10^{15} \mathrm{~s}^{-1}$ (for a virtual intermediate state). On the right-hand side of Eq. (9) the term $\sigma_{1} \Phi(t) n_{1}(t)$ represents absorption leading to a $1 \rightarrow 2$ transition; $\sigma_{1} \Phi(t) n_{2}(t)$ and $k n_{2}(t)$ are the stimulated and spontaneous emission terms, respectively, leading to a $2 \rightarrow 1$ transition; and $\sigma_{2} \Phi(t) n_{2}(t)$ is the absorption term leading to a $2 \rightarrow 3$ transition (into the ionized state). We take $n_{1}(t)+n_{2}(t)+n_{3}(t)$ $=1$, which means that we consider relative populations or, equivalently, that we calculate probabilities. The laser pulse is taken to be rectangular, i.e., $\Phi(t)=\Phi$ for $0 \leqslant t \leqslant \tau$, and $\Phi(t)=0$ at all other times. The boundary conditions then obviously have to be $n_{1}(t=0)=1$ and $n_{2,3}(t=0)=0$. The ionization probability at the end of the pulse is given by $n_{3}(t=\tau)$; we will write $P(\Phi)$ for this quantity to emphasize its intensity dependence. For the duration of the rectangular pulse we took $\tau=\int F\left(t^{\prime}\right) d t^{\prime}=532.2 \mathrm{fs}$, so that it mimics a Gaussian pulse profile with a FWHM duration of 500 fs that has the same pulse energy.

The general solution of Eq. (9) can be written in a compact way as

$$
\begin{aligned}
P(\Phi)= & 1+\frac{1}{2}(\sec B-1) \exp \left(-\frac{A}{2}(\cos B+1)\right) \\
& -\frac{1}{2}(\sec B+1) \exp \left(-\frac{A}{2}(\cos B-1)\right),
\end{aligned}
$$

with $A=\left[k+\left(2 \sigma_{1}+\sigma_{2}\right) \Phi\right] \tau$ and $B=\arcsin \left(2 \sqrt{\sigma_{1} \sigma_{2}} \Phi \tau /\right.$ $A)$. For $\Phi \rightarrow 0$ the ionization probability is given by

$$
P(\Phi)=\sigma_{1} \sigma_{2} \frac{\Phi^{2}}{k^{2}}\left(\mathrm{e}^{-k \tau}+k \tau-1\right),
$$

which for the resonant case (i.e., $k \tau \rightarrow 0$ ) reduces to the fluence-dependent expression

$$
P(\Phi)=\frac{1}{2} \sigma_{1} \sigma_{2}(\Phi \tau)^{2},
$$

whereas for the nonresonant case (i.e., $k \tau \gg 1$ ) the ionization probability is intensity dependent according to

$$
P(\Phi)=\sigma_{1} \sigma_{2} \Phi^{2}\left(\frac{\tau}{k}\right) .
$$

Defining a generalized two-photon cross section $\sigma_{(2)}$, we can summarize the low-intensity rates of Eqs. (12) and (13) as $[10] P(\Phi) / \tau=\left(\sigma_{1} \Phi\right) \tau_{\text {int }}\left(\sigma_{2} \Phi\right)=\sigma_{(2)} \Phi^{2}$, in which $\sigma_{1} \Phi$ is the transition rate going from the ground state to the intermediate state, $\tau_{\text {int }}$ is an effective lifetime of the intermediate state, and $\sigma_{2} \Phi$ is the transition rate going from the intermediate state to the ionized state. Evidently, $\sigma_{(2)}$ can always be expressed as $\sigma_{1} \tau_{\text {int }} \sigma_{2}$ if we choose the effective lifetime $\tau_{\text {int }}$ of the intermediate state to be $k^{-1}$ for a nonresonant process and $\frac{1}{2} \tau$ (i.e., half the effective temporal width of the laser pulse) for a resonant process. Taking $k=10^{15} \mathrm{~s}^{-1}$ for a virtual state, we see that for equal cross sections a resonant process is two to three orders of magnitude more effective than a nonresonant process for our laser pulse duration. 
Interestingly, Eqs. (12) and (13) show that for both a resonant and a nonresonant process the ion-yield curve will always have a quadratic intensity dependence for sufficiently low intensities. This means that the only information that can be deduced from measurements in this intensity range is the fact that a two-photon process occurs. As explained above, one would have to vary the pulse duration to discriminate between resonant and nonresonant processes. However, with our special time-of-flight spectrometer that has a confined source volume, we can unambiguously define an absolute ionization probability if we can reach the saturation plateau, since for those intensities $P(\Phi)=1$, so that we can normalize our measured curves accordingly. In addition, as we will demonstrate below for the case of $\mathrm{Cd}$, this advanced technique allows us to discriminate between a resonant and a nonresonant process without varying the pulse duration.

To explain this now in somewhat more detail, we give the approximation of Eq. (10) for the nonresonant case (i.e., for $k \tau \gg 1, k \gg \sigma_{1} \Phi$, and $\left.k \gg \sigma_{2} \Phi\right)$ :

$$
P(\Phi)=1-e^{-k \tau \sigma_{1} \sigma_{2}(\Phi / k)^{2}}=1-e^{-\left(\Phi / \Phi_{\mathrm{sat}}\right)^{2}},
$$

where the saturation flux is given by $\Phi_{\mathrm{sat}}=\sqrt{k / \sigma_{1} \sigma_{2} \tau}$; we have thus found Eq. (4) again, as expected. For the resonant case (i.e., for $k \tau \rightarrow 0$ ) we find (cf Ref. [18])

$$
\begin{aligned}
P(\Phi)= & +\frac{e^{-\left(s_{1}+s_{2}\right) \Phi \tau / 2}\left(s_{1}-s_{2}\right)}{2 s_{2}} \\
& -\frac{e^{-\left(s_{1}-s_{2}\right) \Phi \tau / 2}\left(s_{1}+s_{2}\right)}{2 s_{2}},
\end{aligned}
$$

where for compactness we have used the abbreviations $s_{1}$ $=2 \sigma_{1}+\sigma_{2}$ and $s_{2}=\sqrt{4 \sigma_{1}^{2}+\sigma_{2}^{2}}$. Since absolute values for the parameters $\Phi$ and $\tau$ are experimentally known, we can now fit curves calculated using Eq. (14) or (15) to our measured data and obtain absolute values for the fit parameters. These fit parameters are $\Phi_{\text {sat }}$ for the nonresonant case and $\sigma_{1}$ and $\sigma_{2}$ for the resonant case. As a result of the symmetry contained in Eq. (15), for every fitted pair $\left(\sigma_{1}, \sigma_{2}\right)=(a, b)$ there exists another pair $\left(\sigma_{1}, \sigma_{2}\right)=\left(\frac{1}{2} b, 2 a\right)$ that yields exactly the same $P(\Phi)$ curve. This implies that the one-photon bound-bound cross section $\sigma_{1}$ cannot be unequivocally determined as two interpretations are possible, namely, $\sigma_{1}=a$ or $\sigma_{1}=\frac{1}{2} b$. In Figs. 1-6 fitted curves based on Eq. (14) or (15) are shown as solid lines. In some cases a reasonable model fit can be obtained even though the presence of molecules is obvious (see the data for $\mathrm{Cu}, \mathrm{Nb}, \mathrm{Gd}, \mathrm{Tb}$, and $\mathrm{Lu}$ in Figs. 1-6). This might be an indication that sputtered atoms are the dominant ion source.

In Fig. 8 we show some typical calculated example curves based on Eqs. (14) and (15). The solid curve is a nonresonant ionization curve based on Eq. (14), with $\Phi_{\text {sat }}=1$ arbitrary units. The other curves are ion-yield curves based on Eq. (15) for a resonantly enhanced MPI scheme, where the parameters $\sigma_{1}$ and $\sigma_{2}$ were chosen so that the curves have the same asymptotic behavior in the low- and high-intensity limits as the nonresonant curve. The ratio $\sigma_{2} / 2 \sigma_{1}$ that determines the shape of the curves was varied between 1 and 0.0001. As these examples clearly demonstrate, an

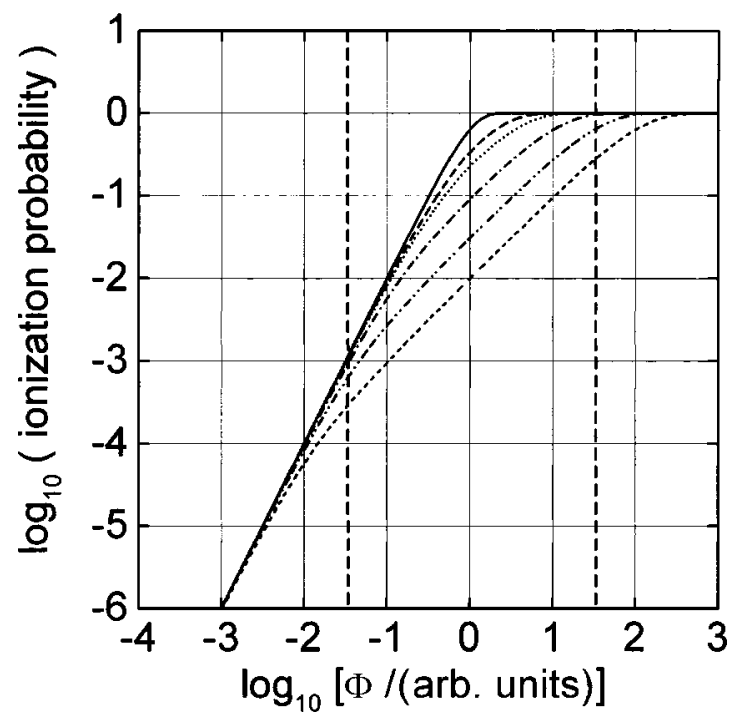

FIG. 8. Calculated example curves showing resonant and nonresonant MPI yields as a function of intensity ( $\tau=22000$ a.u.). The solid curve is calculated based on Eq. (14) and is valid for nonresonant ionization; the corresponding saturation flux $\Phi_{\text {sat }}$ equals the arbitrary unit used on the abscissa. The other curves are ion-yield curves based on Eq. (15) for resonant ionization, where we chose $\sigma_{1}$ and $\sigma_{2}$ so that the curves have the same asymptotic behavior as the solid curve in the low- and high-intensity limits. The following values for the ratio $\sigma_{2} / 2 \sigma_{1}$ were chosen: dashed curve, $\sigma_{2} / 2 \sigma_{1}$ $=1$; dotted curve, 0.1; dash-dotted curve, 0.01; dash-doubledotted, 0.001; short-dashed curve, 0.0001. For the intensity range that is approximately given (for $\sigma_{1} \gg \sigma_{2}$ ) by $1 / \sigma_{1} \tau<\Phi<1 / \sigma_{2} \tau$ a unity slope occurs. The dashed vertical lines approximately indicate the dynamic range in intensity we can cover in the experiment (the position of this range with respect to the curves is arbitrary).

intermediate-intensity range with unity slope (linear intensity dependence) occurs when the two one-photon cross sections $\sigma_{1}$ and $\sigma_{2}$ are very different and one of the two steps is saturated. The dynamic range in intensity that we can cover in our experiments is approximately indicated by the two vertical dashed lines. In our measurements, of course, the relative position of this "window" and the curve to be measured depends on the values of the one-photon cross sections $\sigma_{1}$ and $\sigma_{2}$.

To put further emphasis on the differences between resonant and nonresonant ionization, we have drawn the limiting resonant curve with $\sigma_{2}=2 \sigma_{1}$ in Fig. 7 as well (dashed curve). Clearly, our measured $\mathrm{Cd}$ data exactly follow the nonresonant curve, accurately reproducing the characteristic sharp twist in the neighborhood of the saturation intensity. With our method we can therefore exclude all possible resonant curves, even the one for $\sigma_{2}=2 \sigma_{1}$, in which case saturation is reached fastest, and the range of intensities for which the unity slope is obtained has shrunk to a minimum. It must be stressed that this conclusion remains unchanged even if we take into account the spatial variation of the intensity inside the confined volume and the $30 \%$ uncertainty in our intensity measurements. The ability of our method to discriminate between a resonant and a nonresonant process without variation of the pulse duration might be of general importance. It is critically dependent on the absence of any 
volume effect that would obscure any difference.

For Sc, Fe, Co, Sm, Dy, Ho, Er, and Ta, we summarize experimentally determined values for $\sigma_{1}$ and $\sigma_{2}$ in Table I. As explained before, it is fundamentally impossible to determine which of the measured cross sections corresponds to the bound-bound step $1 \rightarrow 2$ and which to the bound-free step $2 \rightarrow 3$. To rationalize the measured values we have calculated the dipole cross sections of quasiresonant bound-bound transitions for a selection of atoms. However, before proceeding with these calculations, we want to discuss briefly the second, bound-free, step. This step takes the atoms from an intermediate excited state into the continuum, thus completing the photoionization process. Again, we can discriminate between nonresonant and resonant photoionization. For nonresonant photoionization, a relatively small cross section of $10^{-17}-10^{-19} \mathrm{~cm}^{2}$ is expected. However, the presence of rapidly decaying autoionizing states embedded in the continuum leads to a marked increase of the cross section by one or two orders of magnitude [18-20]. One can anticipate the presence of a Rydberg series of such autoionizing states just below bound states of the ionic species. More specifically, for an ionic bound state with an excitation energy of $E_{b}$ above the ionic ground state, we can expect a resonance with an autoionizing state to occur if a condition such as the simplified

$$
\left(E_{i}+E_{b}\right)\left(1-\frac{1}{n^{2}}\right)=2 \hbar \omega
$$

is fulfilled, where $E_{i}$ is the ionization potential of the neutral atom and $n$ (some large value of) the appropriate principal quantum number describing the Rydberg series. Thus the possible closeness of ionic states to the two-photon level could be an indication of a large cross section for the second step. Indeed, states fulfilling the simplified equation (16) can be found, e.g., for Ta, for which the experimental values for $\sigma_{1}$ and $\sigma_{2}$ are both large $\left(\sim 10^{-15} \mathrm{~cm}^{2}\right)$. Of course, transitions between excited atomic states and autoionizing states are again governed by parity and angular momentum selection rules and a more detailed knowledge of the spectrum of autoionizing states than the one expressed in Eq. (16) would be required for an in-depth analysis. Furthermore, we have to take into account that more than one intermediate excited state can play a role. To pursue this matter in a more thorough way would lead outside the scope of this work and we therefore conclude by mentioning that all of our measured cross sections fall within the expected range.

Let us now return to the calculation of the cross sections for the first, bound-bound, step. In these calculations we have to consider the influence of the linear polarization of our laser. In our description of the absorption process we take the $z$ axis parallel to the polarization direction. The atomic states are designated by $|\gamma, J, M\rangle$, where $J$ and $M$ are the quantum numbers of the total angular momentum and its component in the $z$ direction, respectively, and all other quantum numbers are represented by $\gamma$. Since the laser bandwidth $\Delta \omega_{L}$ of about $160 \mathrm{~cm}^{-1}$ is very large in comparison to a typical Doppler width of $1 \mathrm{~cm}^{-1}$ of a dipole-allowed bound atomic state [21], we have broadband excitation, so that the cross section for the bound-bound transition $\sigma_{1}$ $=\sigma\left(|\gamma, J, M\rangle \rightarrow\left|\gamma^{\prime}, J^{\prime}, M^{\prime}\right\rangle\right)$ can be written as [22]

$$
\begin{aligned}
\sigma\left(|\gamma, J, M\rangle \rightarrow\left|\gamma^{\prime}, J^{\prime}, M^{\prime}\right\rangle\right) & \\
= & \varphi_{L}\left(\omega_{0}\right) \frac{4 \pi^{2} \alpha \omega_{0}}{e^{2}}\left|\left(\begin{array}{ccc}
J & 1 & J^{\prime} \\
-M & 0 & M^{\prime}
\end{array}\right)\right|^{2} \\
& \times\left|\left(\gamma J\|\mathrm{D}\| \gamma^{\prime} J^{\prime}\right)\right|^{2},
\end{aligned}
$$

where we have used the Wigner $3 j$ symbol and $\alpha$ is the fine-structure constant, $e$ is the electron charge, $\omega_{0}$ is the center frequency of the atomic transition, and $\left(\gamma J\|\mathrm{D}\| \gamma^{\prime} J^{\prime}\right)$ is a reduced matrix element of the dipole operator D. Furthermore, $\varphi_{L}(\omega)$ is the relative spectral intensity of the laser, normalized according to $\int d \omega \varphi_{L}(\omega)=1$. Measurements of this spectral intensity distribution using a diode array show that it has an exponential decay in the wings and a somewhat complicated structure around the center frequency (see also Ref. [8]). In our calculations we did not use this experimental curve, but instead employed a more practical representation for $\varphi_{L}(\omega)$ that does have the experimentally observed exponential decay in the far wings but has a smooth behavior around the center frequency. This simplification introduces an error in the calculated cross sections of less than a factor of 2. Because of the exponential decay in the wings, the cross section strongly depends on the detuning $\delta=\omega_{0}$ $-\omega_{L}$.

From Eq. (17) we can immediately derive the appropriate selection rules $\left(\Delta J=0\right.$ or $\pm 1, \Delta M=0$, and $\left.J+J^{\prime} \geqslant 1\right)$ that apply in addition to the parity selection rule. In addition, Eq. (17) shows that the magnetic substates of a degenerated atomic ground state are not all depopulated with the same rate since for different values of $M=M^{\prime}$ the $3 j$ symbol takes on different values. For the particular case of a $\mid \gamma, J, M$ $=0\rangle \rightarrow\left|\gamma^{\prime}, J^{\prime}=J, M^{\prime}=0\right\rangle$ transition, the rate is zero because the corresponding $3 j$ symbol in Eq. (17) is zero. In our rate model we did not take into account any degeneracy. If, for a particular transition, the value of the $3 j$ symbol happens to vary dramatically for different values of $M$, we would in fact have different species of neutral atoms and then the total yield must be calculated as the sum of the yields for each particular species. If, however, the variation among the $3 j$ symbols for a single transition is less than the typical experimental uncertainty of $30 \%$ in our intensity measurements one would expect the single cross section rate model to be directly applicable. The reduced matrix element in Eq. (17) can easily be calculated from tabulated values [14] of the oscillator strengths $g f \equiv\left(2 J_{1}+1\right) f_{12}$ for $|\gamma, J\rangle \rightarrow\left|\gamma^{\prime}, J^{\prime}\right\rangle$ transitions through [23]

$$
\left|\left(\gamma J\|\mathrm{D}\| \gamma^{\prime} J^{\prime}\right)\right|^{2}=\frac{3 \hbar e^{2}}{2 m} \frac{1}{\omega_{0}}(g f) .
$$

Combining Eqs. (17) and (18) and dropping the $3 j$ symbol for a moment, we find, in practical units,

$$
\sigma_{1} \approx 1800 \frac{\varphi_{L}\left(\omega_{0}\right)}{\varphi_{L}\left(\omega_{L}\right)}(g f) \times 10^{-17} \mathrm{~cm}^{2},
$$

indicating that in the case of exact resonance $\left(\omega_{0}=\omega_{L}\right)$ the cross section can be as large as $10^{-14} \mathrm{~cm}^{2}$ for an oscillator strength of $g f \approx 1$ and that for the more frequently encountered value of $g f \approx 10^{-3}$ a value of about $10^{-17} \mathrm{~cm}^{2}$ is 
found. Indeed, our measurements show exceptionally large cross sections for $\mathrm{Fe}$ and $\mathrm{Ta}$ and these two atoms have exceptionally large oscillator strengths at the laser excitation frequency.

A complicating factor in our considerations is the role of excited states. Excited states can be populated as a result of the sputtering process, although the majority of the emitted particles are neutral and in the ground state. The formation of excited-state atoms is very sensitive to the chemical environment [16]. In many metal atoms, the ground state is part of a multiplet, with the first few excited states lying typically within the first few $1000 \mathrm{~cm}^{-1}$. All states of the multiplet have the same parity as the ground state. This will inhibit rapid radiative decay to the ground state, so that atoms that were brought into such low-lying excited states can arrive intact in the confined source volume, where they are ionized. (A mass 100 particle with 5-eV kinetic energy would travel the distance of $1 \mathrm{~mm}$ from the target surface to the confined ionization volume in $0.32 \mu \mathrm{s}$.) If a considerable fraction of the sputtered atoms would be in such a metastable excited state and if this excited state would have a bound-bound transition cross section that is very different from that of the ground state, the ionization would be the result of two independent processes, which might lead to the formation of a knee in the ion-yield curve. Furthermore, also the second, bound-free, transition cross section may depend on the intermediate state involved, which in turn will depend on the initial state. Scenarios like this could in principle explain the striking intermediate plateau in the $\mathrm{Al}^{+}$curve. However, as we will demonstrate below, the spectroscopy of the $\mathrm{Al}$ atom does not permit such an explanation. In fact, in this case we see a dimer ion signal as well and the presence of molecular species might make the picture more complicated. For In and, more clearly, for $\mathrm{Yb}$ we observe a kind of knee in the ion-yield curve, but in both cases it is followed by a slight but significant reduction in the ion yield that cannot be explained by metastable states either. It should be noted that this decrease in the singly charged ion takes place when the doubly charged ion becomes observable. The overall trend, however, of the $\mathrm{In}^{+}$yield is still properly described by our rate model as the solid curve in the In graph (see Fig. 3) demonstrates.

Apart from being directly sputtered off the surface, metastable states can also be populated as a result of an allowed decay of higher-lying states. Since we do not have any information on excited-state populations, we have to consider the entire ground-state multiplet of each atom for a realistic calculation of cross sections. Below, this is exemplified for $\mathrm{Sc}, \mathrm{Fe}, \mathrm{Co}$, and $\mathrm{Ta}$, for which we calculated cross sections using Eqs. (17) and (18). We take these four atoms as test cases because their ion yield curves are very smooth, they show typical $(1+1)$ REMPI behavior and no dimers are observed.

We remark that the same holds for the lanthanides Sm, Dy, Ho, and Er, but for these atoms the ionization potentials are so low that the first photon falls in a region of Rydberg states, where the assumption of a single resonant state cannot be valid. Photoionization of $\mathrm{Ni}$ is also a resonant process. This is clearly indicated by the fact that for increasing intensities the $\mathrm{Ni}^{+}$curve gradually changes slope from two to one (see the dashed lines in Fig. 1), with a twist around 1.0 $\times 10^{10} \mathrm{~W} \mathrm{~cm}^{-2}$. Unfortunately, in our Ni measurements, the highest employed intensity was only $1.5 \times 10^{11} \mathrm{~W} \mathrm{~cm}^{-2}$, which was apparently not sufficient to cause saturation. This also explains why the doubly charged ion, which for many atoms has an appearance intensity of about $10^{11} \mathrm{~W} \mathrm{~cm}^{-2}$, was not observed for $\mathrm{Ni}$.

The ground-state multiplets of our four test case atoms are as follows. For Sc, there are two sublevels: $a^{2} D_{J}$, with $J$ $=\frac{3}{2}, \frac{5}{2}$. For Fe, there are five sublevels: $a^{5} D_{J}$, with $J$ $=4,3,2,1,0$. For Co, there are four sublevels: $a^{4} F_{J}$, with $J$ $=\frac{9}{2}, \frac{7}{2}, \frac{5}{2}, \frac{3}{2}$. Finally, for Ta, there are four sublevels: $a^{4} F_{J}$, with $J=\frac{3}{2}, \frac{5}{2}, \frac{7}{2}, \frac{9}{2}$. In each of these lists, the first $J$ value corresponds to the ground state. (Fe and $\mathrm{Co}$ have inverted ground-state multiplets.) In our calculations, we first tried to find all allowed transitions that are located within a few 100 $\mathrm{cm}^{-1}$ around the laser frequency [the exponential decay of $\varphi_{L}(\omega)$ in its wings sidelines allowed transitions that are detuned outside this range], starting from any state in the ground-state multiplet. Then, for each of these transitions, we calculated the corresponding cross section using Eqs. (17) and (18). We thus arrived at the following conclusions. For $\mathrm{Fe}$, the ground state and the first excited state both have a cross section of about $300 \times 10^{-17} \mathrm{~cm}^{2}$ and all the other three states of the multiplet have cross sections of more than $100 \times 10^{-17} \mathrm{~cm}^{2}$ (see also Table I). Therefore, whatever the distribution of the $\mathrm{Fe}$ atoms over this ground-state multiplet, one will always encounter a large cross section for the bound-bound step. The calculated large cross sections are in excellent agreement with the experimentally determined $(460 \pm 240) \times 10^{-17} \mathrm{~cm}^{2}$ (see Table I). (This cross section is so large that a slope larger than one is hardly observable in our experimental curves. In other words, the intensity in our experiments was never low enough to leave the bound-bound step unsaturated.) For Co, the situation is different. Here the largest possible cross section starting from the ground state is on the order of only $10^{-24} \mathrm{~cm}^{2}$, a value flagrantly inconsistent with the experimental values that are both on the order of $10^{-17} \mathrm{~cm}^{2}$ (see Table I). However, starting from the $a^{4} F_{3 / 2}$ state, which lies only $1809.33 \mathrm{~cm}^{-1}$ above the ground state, we obtain very reasonable values of $2.5 \times 10^{-17} \mathrm{~cm}^{2}$ (for $M= \pm \frac{1}{2}$; see Table I) or $1.5 \times 10^{-17} \mathrm{~cm}^{2}$ (for $M=$ $\left.\pm \frac{3}{2}\right)$. For the other states of the ground-state multiplet, we calculated cross sections that are at least 15 times smaller. We thus have to conclude that for $\mathrm{Co}$ all atoms that were created in the ground state remain unionized and that the measured ionization signal originates almost exclusively from those atoms that were brought into the $a^{4} F_{3 / 2}$ excited state. For all other states of the ground-state multiplet, the cross sections are negligibly small. Assuming equal detection efficiencies for $\mathrm{Fe}^{+}$and $\mathrm{Co}^{+}$(their atomic masses $M_{\mathrm{Fe}}$ $=56$ and $M_{\mathrm{Co}}=59$ are similar) we can compare the ratio between our measured "saturated" $\mathrm{Co}^{+}$yield $Y_{\mathrm{Co}}$ and our measured saturated $\mathrm{Fe}^{+}$yield $Y_{\mathrm{Fe}}$ to literature values [24] of sputtering yields. The sputtering yield is defined as the ratio of the number of ejected to incoming atoms. Our measured ratio $Y_{\mathrm{Co}} / Y_{\mathrm{Fe}}$ is only about $8 \%$ of the literature value. Assuming that we do saturate Fe, this confirms our suspicion that only metastable Co atoms contribute to our measured $\mathrm{Co}^{+}$signal.

The next test case atom is Sc. Here, starting from the ground state, one finds, e.g., $1.2 \times 10^{-18} \mathrm{~cm}^{2}$ for $M= \pm \frac{3}{2}$, 
but starting from the first excited state a larger value is found (e.g., $10 \times 10^{-18} \mathrm{~cm}^{2}$ for $M= \pm \frac{1}{2}$; see Table I). Apparently, our $\mathrm{Sc}^{+}$signal originates exclusively from the excited state, as the experimentally determined bound-bound cross section of $(16 \pm 8) \times 10^{-18} \mathrm{~cm}^{2}$ fits very well with the theoretical value of the excited state (see Table I). Substantial ionization starting from the ground state is expected to occur only for intensities beyond $10^{12} \mathrm{~W} \mathrm{~cm}^{-2}$, where, unfortunately, no data points are available. Using a theoretical value [24] for the sputtering yield of Sc, we estimate our maximum ion signal to be only $7 \%$ of a fully saturated value, again in qualitative agreement with our calculations. Finally, we will discuss the example of Ta. For this atom, again taking into account just the ground-state multiplet, a large cross section of $65 \times 10^{-17} \mathrm{~cm}^{2}$ is found for the $M= \pm \frac{3}{2}$ substates of the $a^{4} F_{3 / 2}$ ground state, which contain $50 \%$ of all ground state atoms (see also Table I). The cross section for the other 50\%, which is in the $M= \pm \frac{1}{2}$ substates, is about 9 times smaller. For all other states of the ground-state multiplet, the calculated cross sections are at least 20 times smaller, with the exception of the $a^{4} F_{7 / 2}$ state, which has a cross section of $32 \times 10^{-17} \mathrm{~cm}^{2}$ (for $M= \pm \frac{7}{2}$ ). Assuming that the majority of the $\mathrm{Ta}$ atoms were created in the ground state, we would expect the following picture for the $\mathrm{Ta}^{+}$yield curve: After reaching an intermediate plateau extending over about one order of magnitude in intensity, the yield would increase again by a factor of 2 and then reach the final plateau of $100 \%$ ionization. Interestingly, this is what one finds by adding the $\mathrm{Ta}^{+}$and $\mathrm{Ta}^{2+}$ yields, if one first divides the $\mathrm{Ta}^{2+}$ yields by a factor of 2 , which takes into account that the detection efficiency for $\mathrm{Ta}^{2+}$ is higher than for $\mathrm{Ta}^{+}$. Unfortunately, our detection efficiencies can only be estimated, but the final saturated ion yield if we use a factor of 2 matches exactly the expectations based on sputtering yields [24]. In any case, the theoretical value for the $a^{4} F_{3 / 2}\left(M= \pm \frac{3}{2}\right)$ cross section of $65 \times 10^{-17} \mathrm{~cm}^{2}$ agrees very well with our experimental value of $\left(30_{-15}^{+80}\right) \times 10^{-17} \mathrm{~cm}^{2}$ that we obtained from curve fitting below $10^{11} \mathrm{~W} \mathrm{~cm}^{-2}$.

These four test cases demonstrate that detailed knowledge about the population of low-lying excited states is an important precondition if one wants to compare calculated cross sections to measured ones. Furthermore, as the examples of $\mathrm{Co}$ and $\mathrm{Sc}$ show, it is by no means certain that the observation of a saturation plateau in an ionization signal implies that all neutral atoms are ionized. This important fact has drastic implications for the application of the SALI technique for quantitative surface analysis. The test cases further indicate that experimental and theoretical values are in reasonable agreement.

\section{Some final remarks}

We do not observe saturation for Mg. For this atom (as for $\mathrm{Zn}$ and $\mathrm{Cd}$ ), no (quasi)resonant intermediate state exists. (Remarkably, it is for these three atoms that we do not observe higher charge states.) As explained above, the observations for $\mathrm{Zn}$ and $\mathrm{Cd}$, both being nonresonant systems, are in agreement with the scaling theory, which is known to generate overestimated saturation intensities. The situation for $\mathrm{Mg}$ is surprising, as one would expect an ion yield curve very similar to the $\mathrm{Zn}$ and $\mathrm{Cd}$ curves. For $\mathrm{Mg}$, no saturation occurs up to the highest employed intensity of 1.5 $\times 10^{12} \mathrm{~W} \mathrm{~cm}^{-2}$, although the scaling theory predicts the saturation intensity of $\mathrm{Mg}\left(8.0 \times 10^{12} \mathrm{~W} \mathrm{~cm}^{-2}\right)$ to be even lower than that of $\mathrm{Cd}\left(1.0 \times 10^{13} \mathrm{~W} \mathrm{~cm}^{-2}\right)$, which we could clearly saturate. Certainly, the saturation intensity predicted for $\mathrm{Mg}$ by the scaling law is still beyond the maximum intensity employed in our $\mathrm{Mg}$ measurements and the $\mathrm{Mg}$ ions do appear relatively "late," but the fact that for $\mathrm{Mg}$ we do not find a slope of 2 as we did for $\mathrm{Zn}$ and $\mathrm{Cd}$ is in conflict with a nonresonant two-photon-ionization picture. The curve for W has a slope of about one. Unfortunately, however, we only recorded the $\mathrm{W}$ curve over a narrow intensity range, so that one does not see a clear twist in the ion curve as is the case for $\mathrm{Ni}$, where we recorded over an intensity range that is about an order of magnitude wider. (Certainly, the discernible twist in the $\mathrm{W}$ curve around $5 \times 10^{10} \mathrm{~W} \mathrm{~cm}^{-2}$ is different in nature than the twist in the $\mathrm{Ni}$ curve since in the $\mathrm{W}$ curve the slope for intensities beyond the twist is much less than one.) The ion-yield curve for $\mathrm{Rh}(Z=45)$ seems to follow a slope of 2 for intensities below $4 \times 10^{10} \mathrm{~W} \mathrm{~cm}^{-2}$, but beyond that intensity the curve quite abruptly changes slope to about one. However, even for the highest intensities employed, up to $10^{12} \mathrm{~W} \mathrm{~cm}^{-2}$, there is no indication of saturation. The same holds for $\operatorname{Re}(Z=75)$. Between $1 \times 10^{10}$ and $4 \times 10^{11} \mathrm{~W} \mathrm{~cm}^{-2}$ the slope of the $\mathrm{Tm}^{+}$curve decreases smoothly, but no real saturation is observed. For all these three atoms, the curve does not resemble the nonresonant $\mathrm{Cd}$ curve and for $\mathrm{Rh}$ and $\mathrm{Tm}$ one can in fact find quasiresonant allowed transitions. However, this being so, why is no saturation observed, not even for intensities beyond our experimental saturation intensity of $\mathrm{Cd}$, which undergoes nonresonant ionization? The nonresonant saturation intensities predicted by the scaling model for $\mathrm{Rh}, \mathrm{Tm}$, and Re are comparable to or smaller than the $\mathrm{Cd}$ value. Our measured In $(Z=49)$ curve can be reasonably reproduced by our rate model for most intensities, but it exhibits a slight decrease in the ion yield around $2 \times 10^{11} \mathrm{~W} \mathrm{~cm}^{-2}$ that cannot be explained within this model. A similar small reduction in ion yield is found for $\mathrm{Yb}$. Remarkably, for both In and $\mathrm{Yb}$ the doubly charged ion becomes detectable at the intensity where the knee occurs. Finally, we will discuss the Al curve again. The ground state of $\mathrm{Al}$ is part of a doublet termed $3{ }^{2} P_{J}$, with $J=\frac{1}{2}$ (for the ground state) or $J=\frac{3}{2}$ (for the excited state at $112.04 \mathrm{~cm}^{-1}$ ). Starting from this doublet, no resonant intermediate state exists. The next excited state that has the same parity as the ground-state multiplet is found in the $4 p{ }^{2} P_{J}\left(J=\frac{1}{2}, \frac{3}{2}\right)$ multiplet, which is more than $4 \mathrm{eV}$ away. If ionization would take place from there, it would no longer be a two-photon process because the ionization potential of $\mathrm{Al}$ amounts to only $5.984 \mathrm{eV}$. The initial slope of the $\mathrm{Al}$ ion curve is significantly larger than one and can only be explained as the result of an additional process starting not from the neutral atom but from some molecular species created in the sputtering process. Between $10^{10}$ and 2 $\times 10^{10} \mathrm{~W} \mathrm{~cm}^{-2}$ this process is saturated, giving rise to a plateau for higher intensities. For intensities beyond 3 $\times 10^{11} \mathrm{~W} \mathrm{~cm}^{-2}$, another process becomes dominant and leads to additional production of the singly charged ion. Because the slope of the curve at the highest intensities is close to 2, one may speculate that this latter process is in fact nonresonant ionization, rapidly depleting the neutral ground- 
state atom that remained unaffected thus far. The scaling law predicts a saturation intensity of $7.8 \times 10^{12} \mathrm{~W} \mathrm{~cm}^{-2}$ for nonresonant MPI of $\mathrm{Al}$, not at variance with the present measurements.

\section{CONCLUSIONS}

Using a powerful combination of ion-beam sputtering and laser postionization techniques in conjunction with an advanced time-of-flight detection method that is absolutely free of volume effects, we have measured ion yield curves as a function of laser intensity for 31 metal atoms. We have demonstrated that this method allows us to discriminate between resonant and nonresonant MPI without varying the pulse duration. For resonantly enhanced MPI we have employed a rate equation model that reproduces the experimental ionyield curves for the singly charged species very well and by curve fitting we have determined absolute experimental values for the one-photon cross sections appearing in this model. For a selection of atoms we have also calculated the excitation cross section of the first (bound-bound) step of our two-step model and the agreement between theoretical and experimental results is good. For the linear polarization employed in this work the cross sections for different magnetic substates of the atomic ground state may substantially differ among each other. Furthermore, excited metastable states of the atom that were populated as a result of the sputtering process can also have cross sections that are very different from the ground-state cross section. Therefore, one can expect the production of the singly charged ion to be the result of several independent processes and thus one can anticipate several intermediate plateaus to appear in the ion-yield curves for increasing intensities before the actual saturation plateau is reached. We have possibly observed such an intermediate plateau for Ta. Clearly, the creation of atoms in a metastable state can be avoided if one uses thermal evaporation (instead of sputtering) to free them from the solid target.
However, this will leave the differences in cross section among the different magnetic substates of the ground state, so that intermediate plateaus can still be expected. Generally speaking, one expects nonresonant MPI to become dominant over resonant MPI as soon as the nonresonant MPI saturation intensity is approached, so that for the ultrashort pulse duration of $500 \mathrm{fs}$ employed here eventually all neutral atoms will be ionized for intensities of at most $\sim 10^{13} \mathrm{~W} \mathrm{~cm}^{-2}$ (typical largest scaling model value). However, for a practical application of this technique as a quantitative tool for sample surface analysis, one would prefer to work with an easily available common excimer laser that can produce focused intensities similar to the ones employed here. For such a laser the pulse duration is four to five orders of magnitude longer and for nonresonant MPI processes the saturation intensities (which scale with $\tau^{-1 / 2}$ ) will be some 200 times lower. The saturation intensity of resonantly enhanced MPI processes, on the other hand, depends only on the pulse fluence and thus scales with $\tau^{-1}$. We therefore expect saturation intensities on the order of $10^{6}-10^{7} \mathrm{~W} \mathrm{~cm}^{-2}$. This prediction matches the experiments in Ref. [4], where a $\mathrm{KrF}$ excimer laser with a pulse duration of 22 ns was used to ionize $\mathrm{Fe}$ and a saturation intensity on the order of $10^{7} \mathrm{~W} \mathrm{~cm}^{-2}$ was measured.

\section{ACKNOWLEDGMENTS}

The experimental part of this work was carried out in the Ultraviolet Laser Facility (ULF) operating at FORTH-IESL (HCM Contract No. ERB-CHGE-CT920007). We gratefully acknowledge fruitful discussions with Dr. M. Wagner as well as his presence during the initial phase of the experiments. One of us (C.J.G.J.U.) gratefully acknowledges the Alexander von Humboldt-Stiftung for support during part of this work. We would like to thank A. Eglezis for his skillful technical assistance. We acknowledge discussions with $\mathrm{Ch}$. Gebhardt and P. Maragakis.
[1] G. Voronov and N. Delone, JETP Lett. 1, 66 (1965); P. Agostini, G. Barjot, J. F. Bonnal, G. Mainfray, and C. Manus, IEEE J. Quantum Electron. QE-4, 667 (1968).

[2] For SNMS see, e.g., H. Oechsner, Nucl. Instrum. Methods Phys. Res. B 33, 918 (1988).

[3] C. H. Becker and K. T. Gillen, Anal. Chem. 56, 1671 (1984).

[4] M. Wagner and H. Schröder, Int. J. Mass Spectrom. Ion Processes 128, 31 (1993).

[5] H. Schröder, M. Wagner, S. Kaesdorf, and K.-L. Kompa, Ber. Bunsenges. Phys. Chem. 97, 1688 (1993).

[6] H. Schröder, M. Wagner, and S. Kaesdorf, in Excimer Lasers, edited by L. D. Laude (Kluwer Academic, Dordrecht, 1994).

[7] See, e.g., S. Speiser and J. Jortner, Chem. Phys. Lett. 44, 399 (1976).

[8] S. Szatmári and F. P. Schäfer, Opt. Commun. 68, 196 (1988).

[9] P. Simon, H. Gerhardt, and S. Szatmári, Opt. Commun. 71, 305 (1989).

[10] P. Lambropoulos and X. Tang, J. Opt. Soc. Am. B 4, 821 (1987), and references therein; P. Lambropoulos, Comments
At. Mol. Phys. 20, 199 (1987), and references therein.

[11] P. Lambropoulos, Phys. Rev. Lett. 55, 2141 (1985).

[12] Note that the atomic unit of electric field strength is given in SI units by $F_{0}=E_{h} / e a_{0} \approx 5.142 \times 10^{11} \mathrm{~V} \mathrm{~m}^{-1}$ and the atomic unit of intensity by $I_{0}=E_{h} / t_{0} a_{0}^{2} \approx 6.436 \times 10^{19} \mathrm{~W} \mathrm{~m}^{-2}$. In these two expressions we used the atomic units of energy $E_{h}$ $=\alpha m_{\mathrm{el}} c^{2} \approx 4.360 \times 10^{-18} \mathrm{~J} \approx 27.21 \mathrm{eV}, \quad$ length $\quad a_{0}$ $=4 \pi \epsilon_{0} \hbar^{2} / m_{\mathrm{el}} e^{2} \approx 5.292 \times 10^{-11} \mathrm{~m}$, and time $t_{0}=a_{0} / \alpha c$ $\approx 2.419 \times 10^{-17} \mathrm{~s}$. In atomic units, the intensity and electric field strength are related by $I=F^{2} / 8 \pi \alpha$, which is the equivalent of the SI expression $I=\frac{1}{2} \varepsilon_{0} c F^{2}$.

[13] R. R. Freeman, P. H. Bucksbaum, H. Milchberg, S. Darack, D. Schumacher, and G. M. Geusic, Phys. Rev. Lett. 59, 1092 (1987).

[14] In this work we have used the following sources for atomic energy levels and oscillator strengths: C. E. Moore, Atomic Energy Levels As Derived From the Analyses of Optical Spectra, Natl. Bur. Stand. (U.S.) Circ. No. 467 (U.S. GPO, Washington, DC, 1949), Vol. I; ibid. (U.S. GPO, Washington, DC, 1952), Vol. II; ibid. (U.S. GPO, Washington, DC, 1959), Vol. 
III; ibid. (U.S. GPO, Washington, DC, 1971); reissued versions (1971); National Institute of Standards and Technology Atomic Spectroscopic Database (version 1.1, currently accessible on the Internet at URL http://physics.nist.gov/ PhysRefData/contents.html), and references therein; P. L. Smith, C. Heise, J. R. Esmond, and R. L. Kurucz, Atomic spectral line database, from CD-ROM 23 of R. L. Kurucz (currently accessible on the Internet at URL http://cfawww.harvard.edu/amp/data/kur23/sekur.html), and references therein.

[15] S. Kaesdorf, M. Hartmann, H. Schröder, and K.-L. Kompa, Int. J. Mass Spectrom. Ion Processes 116, 219 (1992).

[16] G. Betz and K. Wien, Int. J. Mass Spectrom. Ion Processes 140, 1 (1994).

[17] See, e.g., A. Yariv, Optical Electronics, 4th ed. (Saunders College Publishing, Fort Worth, 1991).

[18] V. S. Letokhov, Laser Photoionization Spectroscopy (Academic, London, 1987).

[19] W. Demtröder, in Applied Laser Spectroscopy, edited by W.
Demtröder and M. Inguscio (Plenum, New York, 1990).

[20] K. Ledingham, in An Introduction to Laser Spectroscopy, edited by D. Andrews and D. Demidov (Plenum, New York, 1995).

[21] The most probable speed $v_{\mathrm{mp}}$ of sputtered atoms of mass $M$ is on the order of $\sqrt{U_{b} / M}$, with $U_{b}$ the heat of sublimation (see Ref. [16]). For $\mathrm{Mg}$, the lightest atom we studied, a typical Doppler width $2 \pi v_{\mathrm{mp}} / \lambda_{0}$ is on the order of $1 \mathrm{~cm}^{-1}$ (taking $\left.U_{b} \approx 5 \mathrm{eV}\right)$.

[22] See, e.g., E. Merzbacher, Quantum Mechanics, 2nd ed. (Wiley, New York, 1970); C. Cohen-Tannoudji, B. Diu, and F. Laloë, Quantum Mechanics (Wiley, New York, 1977), Vol. 2.

[23] See, e.g., I. I. Sobelman, Atomic Spectra and Radiative Transitions, 2nd ed. (Springer, Berlin, 1992).

[24] H. H. Andersen and H. L. Bay, in Sputtering by Particle Bombardment I-Physical Sputtering of Single-Element Solids, edited by R. Behrisch, Topics in Applied Physics Vol. 47 (Springer, Berlin, 1981), and references therein. 